\title{
Thyroid Dysfunction as an Unintended Side Effect of Anticancer Drugs
}

\author{
Francesco Torino, Agnese Barnabei, ${ }^{2}$ Rosamaria Paragliola, ${ }^{3}$ Roberto Baldelli, ${ }^{2}$ \\ Marialuisa Appetecchia, ${ }^{2}$ and Salvatore Maria Corsello ${ }^{3}$
}

Background: Several of the currently used anticancer drugs may variably affect thyroid function, with impairment ranging from modified total but not free concentration of thyroid hormones to overt thyroid disease.

Summary: Cytotoxic agents seem to alter thyroid function in a relatively small proportion of adult patients. Anticancer hormone drugs may mainly alter serum levels of thyroid hormone-binding proteins without clinically relevant thyroid dysfunction. Old immunomodulating drugs, such as interferon- $\alpha$ and interleukin-2, are known to induce variably high incidence of autoimmune thyroid dysfunction. Newer immune checkpoint inhibitors, such as anti-CTLA4 monoclonal antibodies, are responsible for a relatively low incidence of thyroiditis and may induce secondary hypothyroidism resulting from hypophysitis. Central hypothyroidism is a well-recognized side effect of bexarotene. Despite their inherent selectivity, tyrosine kinase inhibitors may cause high rates of thyroid dysfunction. Notably, thyroid toxicity seems to be restricted to tyrosine kinase inhibitors targeting key kinase-receptors in angiogenic pathways, but not other kinase-receptors (e.g., epidermal growth factor receptors family or c-KIT). In addition, a number of these agents may also increase the levothyroxine requirement in thyroidectomized patients.

Conclusions: The pathophysiology of thyroid toxicity induced by many anticancer agents is not fully clarified and for others it remains speculative. Thyroid dysfunction induced by anticancer agents is generally manageable and dose reduction or discontinuation of these agents is not required. The prognostic relevance of thyroid autoimmunity, overt and subclinical hypothyroidism induced by anticancer drugs, the value of thyroid hormone replacement in individuals with abnormal thyrotropin following anticancer systemic therapy, and the correct timing of replacement therapy in cancer patients need to be defined more accurately in well-powered prospective clinical trials.

\section{Introduction}

I N CANCER PATIENTS, abnormalities in thyroid function and thyroid diseases are variably associated with cancer itself, diagnostic procedures, or anticancer treatments. Abnormalities of thyroid hormone synthesis and metabolism as well as of thyrotropin (TSH) levels, more commonly known as "euthyroid sick syndrome," may occur in patients with advanced cancers (1). Diagnostic procedures using iodinated contrast agents can be associated with acute effects on the thyroid, including hyperthyroidism triggering/exacerbation, as in patients with autonomous thyroid nodules or Graves' disease, or transient hypothyroidism (i.e., in patients with Hashimoto's thyroiditis) (2,3). Radiation therapy (RT) can lead to primary hypothyroidism due to direct damage to the thyroid or, indirectly, through hypopituitarism due to brain irradiation $(3,4)$. External cervical RT received during childhood has been associated with thyroid nodules and papillary thyroid cancer later in life (4).

Systemic anticancer treatments include old and newer cytotoxic and hormone drugs, immune system modulators, and targeted drugs that selectively modulate key molecules in cancer progression or immune response to cancer. Many of these agents may variably affect thyroid function with impairment ranging from modified total but not free concentration of thyroid hormones to overt thyroid disease $(5,6)$. Anticancer hormone drugs may alter thyroxine-binding globulin levels, but marginally affect thyroid function (Table 1). Only aminoglutethimide, used to control adrenal, breast, and prostate cancer, is reported to cause hypothyroidism in up to $31 \%$ of patients $(7,8)$.

\footnotetext{
${ }^{1}$ Department of Systems Medicine, Tor Vergata University of Rome, Rome, Italy.

${ }^{2}$ Endocrinology Unit, Regina Elena National Cancer Institute, Rome, Italy.

${ }^{3}$ Endocrinology Unit, Università Cattolica, Rome, Italy.
} 
This review focuses on thyroid function abnormalities and diseases resulting from exposure to nonhormonal anticancer agents currently used for the treatment of adult malignancies.

\section{Search Strategy}

The PubMed and MEDLINE databases were searched for articles published before March 30, 2013. Electronic early-release publications are also included. Only articles published in English were considered. The search terms were "thyroid dysfunction," "hypothyroidism," "thyroid toxicity," "endocrine adverse events," and "autoimmunity," in association with "anticancer drugs," "tyrosine kinase inhibitors," "immune checkpoint inhibitors," "interleukin," and "interferon." Proceedings from the 2000-2012 Conferences of the American Society of Clinical Oncology, the European Society of Medical Oncology, and the Endocrine Society were searched for relevant abstracts.

\section{Thyroid and Cytotoxic Anticancer Agents}

In adult survivors of childhood cancers, endocrine toxicity, including thyroid disorders, is a common long-term complication of cytotoxic treatments (9). Conversely, cytotoxic treatments seem to alter thyroid function in a relatively small portion of adult patients (5). However, few studies prospectively evaluated thyroid dysfunction induced by cytotoxic agents in adult populations. Lomustine, vincristine, and cisplatin have shown in vitro effects on thyroid cells (10). In small series, 5-fluorouracil and L-asparaginase alter levels of thyroid hormone-binding proteins without clinical consequence (11-16). L-asparaginase may cause transient central hypothyroidism as well (17).

Mitotane, the only active agent against adrenocortical cancer, reduced both activity and viability of mouse pituitary TSH-secreting cells (18). In patients treated with mitotane for adrenocortical cancer, a marked reduction in free thyroxine (free T4) without concomitant reduction in free triiodothyronine (free T3) (possibly by influence on deiodinase activity) and without elevation of TSH was found (19). Free T4 levels dropped in the hypothyroid range in most patients and were inversely correlated with mitotane plasma levels (19).

Very few studies evaluated the effects induced by polychemotherapy on thyroid function in cancer patients. Primary hypothyroidism was diagnosed in $15 \%$ of patients with testicular cancer receiving a combination regimen of cisplatin, bleomycin, vinblastine, etoposide, and dactinomycin (20). Increased serum TSH levels were documented in $44 \%$ of patients with Hodgkin disease who received mechlorethamine, vinblastine, procarbazine, and prednisolone (MOPP regimen). However, in this small trial, iodine load during lymphangiography may have contributed to the thyroid abnormalities (21).

When delivered concomitantly with head-neck irradiation, cytotoxic chemotherapy increases the risk of RT-induced primary or central hypothyroidism (22-24). In comparison with RT, cytotoxic chemotherapy (CT) alone does not increase the risk of thyroid cancer (5).

\section{Immunoregulatory Drugs}

\section{Interferon- $\alpha$}

Interferon- $\alpha(\mathrm{IFN}-\alpha)$ is a human recombinant cytokine with direct antiviral and antitumor activity, as well as indirect immune-mediated destruction of cancer cells $(25,26)$. IFN- $\alpha$ is approved for patients affected by melanoma, renal cell carcinoma, AIDS-related Kaposi's sarcoma, some hematologic malignancies, and virus $B / C$ hepatitis (27).

IFN-induced thyroid abnormalities can be classified as autoimmune and nonautoimmune thyroiditis $(28,29)$. Autoimmune IFN-induced thyroiditis (IIT) can manifest as a clinical disease (i.e., Graves' disease or Hashimoto's thyroiditis), or as a subclinical disease, as seen in the presence of thyroid autoantibodies (TAb) without overt thyroid dysfunction ( $20 \%$ of patients). Nonautoimmune IIT can present as destructive thyroiditis, or nonautoimmune hypothyroidism $(28,29)$. This classification proposed for IIT experienced by patients affected by hepatitis appears suitable for cancer patient as well.

The incidence of thyroid abnormalities in patients receiving IFN therapy for solid tumors is $2.4-31 \%$ (30-39), with up to $50 \%$ in some trials $(40,41)$. In patients affected by hematological malignancies, the incidence of IFN-related thyroid dysfunction appeared lower $(42,43)$, with the difference remaining unexplained. Autoimmune IIT mainly occurs in the first few weeks of treatment and, following a brief thyrotoxic phase, may lead to hypothyroidism in close temporal relationship with the appearance of TAb, especially Tg-Ab. Hypothyroidism persists in the majority of patients $(31,44)$, although transient hypothyroidism has also been described (45). Pre-existing thyroid peroxidase antibody (TPO-Ab) considerably increases the risk of hypothyroidism $(38,46-48)$. Thyroid abnormalities may recover with the withdrawal of IFN. In two randomized trials of IFN- $\alpha$ on melanoma patients, the association between the appearance of autoantibodies, including $\mathrm{TAb}$, and improved relapse-free interval was not statistically significant. (49).

Rarely, classical Graves' disease and sometimes Graves' ophthalmopathy may develop (50). These conditions may persist despite the withdrawal of the drug (6).

By binding to its receptors, IFN- $\alpha$ triggers several key immune effects, including an increased expression of MHC class I antigens (HLA) on cells, including thyroid epithelial cells (48), enhanced activity of lymphocytes, macrophages, and natural killer cells $(\mathrm{NK})(51,52)$ and decreased T-regs function $(53,54)$. Notably, the IFN $\alpha$-induced HLA overexpression is associated with activation of cytotoxic T-cells leading to cellular destruction, when lymphocytes are present in the tissue (52). This mechanism was also seen in the thyroid and might explain the worsening of pre-existing subclinical thyroiditis (55). Furthermore, IFN- $\alpha$ can switch the immune response to a Th1 pattern $(56,57)$ with an increased production of interferon- $\gamma$ and interleukin-2 (IL2), two potent proinflammatory cytokines, and of IL-6 $(52,56,57)$. This immune pattern may trigger an autoimmune response. Finally, thyroid cells may be directly damaged by IFN- $\alpha$, a suggested mechanism for destructive thyroiditis $(29,48,58)$. Despite accumulating evidence, the precise mechanisms of IIT remain to be elucidated, especially in cancer patients.

\section{Pegylated IFNa}

The pegylation of IFN $\alpha-2 b$ provides a prolonged plasma half-life of the drug, by decreasing renal clearance $(59,60)$, and improved efficacy in solid tumors and melanoma is reported, compared to unpegylated form $(61,62)$. In the 18991 European 
Organisation for Research and Treatment of Cancer trial evaluating pegylated IFNa (Peg-IFN) versus no treatment in highrisk melanoma patients, autoantibodies, including $\mathrm{TgAb}$, were found in $52 \%$ of patients in the Peg-IFN group, compared with $18 \%$ in the control group (60). Better survival was not associated with the development of autoantibodies when analysis was corrected for guarantee-time bias (Table 1) (63).

\section{Interleukin-2}

IL2 is a cytokine involved in several mechanisms of immune response, including activation of NKs and antigenspecific T-cells. IL2 is approved for the treatment of patients affected by metastatic melanoma and renal cell carcinoma, although its use is currently reduced as more effective and better-tolerated agents are available (6).

Thyroid dysfunction and induction of TAb occur in 10-60\% of cancer patients treated with IL2 alone or in combination with IFN- $\alpha$, lymphokine-activated killer cells, or vaccines (6471). An early phase of presumptive destructive thyrotoxicosis is common, with variable degrees of hyperthyroidism $(68,72-$ 74). Hypothyroidism, mostly associated with TAb, occurs 4-17 weeks after treatment has commenced $(64,65)$ and may be reversible following discontinuation of the drug $(65,72)$.

Various autoimmune mechanisms may be involved in IL-induced thyroid toxicity. IL2 is a potent inducer of proinflammatory cytokines such as IL1, tumor necrosis factor alpha, and IFN- $\gamma$ (75-77). In patients with IL2-induced thyroid diseases, high levels of interferon- $\gamma$ and tumor necrosis factor alpha have been found. These cytokines may trigger autoimmune thyroiditis by enhancing the presentation of HLA-II and associated autoantigens on thyrocytes. Also, IL2 may have a direct effect on thyrocyte function $(78,79)$. Increase in $\mathrm{TAb}$ levels $(64,69)$ and lymphocyte infiltration of the thyroid gland $(73,80)$ were found in patients treated with IL2. Similar to IFN- $\alpha$, high TAb levels before treatment increase the risk of IL2-induced hypothyroidism (65). Whether occurrence of hypothyroidism during the treatment with IL2 predicts favorable outcomes remains uncertain (Table 1) $(64,65,81-85)$.

\section{Denileukin diftitox}

In Denileukin diftitox (DD), the ligand-binding domain of IL2 is fused to diphtheria toxin (86). The drug binds to the IL2 receptors on lymphocytes and macrophages and the toxin inhibits protein synthesis, leading to cell death. It is approved for use in cutaneous T-cell lymphoma and graft-versus-host disease after allogenic stem cell transplant $(86,87)$. In a retrospective study, eight patients showed TPO-Ab and thyrotoxicosis within 1-35 days of treatment; five of them eventually developed permanent hypothyroidism (88). Autoimmunity could not be the mechanism of DD-induced thyroid toxicity, because the IL2-component of the drug does not activate the IL2-lymphocyte receptor. The absence of IL2receptors on thyrocytes excludes direct thyroid toxicity (88). However, DD might target and lyse intrathyroidal lymphocytes with local release of cytokines leading to thyroiditis/ thyrotoxicosis (88).

\section{Thalidomide and lenalidomide}

Thalidomide and its derivative lenalidomide are immunomodulatory agents active against multiple myeloma (MM).
Lenalidomide is also efficacious in patients with the $5 q$ myelodysplastic syndrome $(89,90)$. Anticancer activity of these drugs is based on immunomodulatory and antiangiogenic effects and is elicited by NK-mediated cytotoxicity, inhibition of proinflammatory cytokines, and increase in antiinflammatory cytokines secretion and production of proangiogenic factors (e.g., vascular endothelial growth factor [VEGF], basic-fibroblast growth factor, tumor necrosis factor alpha, interleukin-6) (91). Subclinical hypothyroidism was reported in $20 \%$ of patients who received thalidomide for MM with another $7 \%$ of overt hypothyroidism, mostly occurring 1-6 months after treatment has begun (92). In a retrospective study, lenalidomide induced thyroid abnormalities in $6 \%$ of patients affected by MM and increased the risk of progression of thyroid dysfunction in patients with previous thyroid abnormalities (93). In another study, lenalidomide-induced hypothyroidism was found in $5-10 \%$ of patients $(94,95)$. No thyroid abnormalities were reported in a large randomized study on 459 patients with untreated MM, receiving lenalidomide in combination with cytotoxics and prednisone (96).

Mechanisms of thalidomide/lenalidomide-induced hypothyroidism remain uncertain. Interference with thyroid hormone secretion and reduction of iodine uptake into follicular cells have been suggested $(97,98)$. As thalidomide/lenalidomide exert antiangiogenic activity, a compromised thyroid blood flow may trigger thyroid toxicity (92). In some patients, TSH suppression preceded hypothyroidism, suggesting destructive thyroiditis, presumably induced by ischemia $(99,100)$. Alternatively, an immune-mediated destructive thyroiditis may be induced by altered cytokine levels or through direct effects on T-lymphocytes (92).

\section{Immune checkpoint inhibitors}

Recent progress in cancer immunotherapy led to the development of immune regulatory monoclonal antibodies $(\mathrm{MAb})$ that inhibit immunologic checkpoints, such as the cytotoxic T-lymphocyte antigen-4 receptor (CTLA-4) and the programmed death-1 receptor pathway (101). The anticancer activity of these drugs is presumably obtained by unleashing tumor immune tolerance $(102,103)$. Ipilimumab and tremelimumab are immunomodulating $\mathrm{MAb}$ directed against CTLA-4 (anti-CTLA4-MAb), which have shown variable activity against several malignancies (104). Ipilimumab is approved for clinical use in patients with advanced cutaneous melanoma (105). However, inhibition of CTLA-4 induces a series of immune-related adverse events (106-108), mainly colitis/diarrhea, dermatitis, hepatitis, and endocrinopathies (109). Among endocrine toxicities, hypophysitis has emerged as a distinctive side effect of anti-CTLA4-MAb $(106,110)$. As the damage to TSH-secreting cells and corticotroph is prevalent (111), in patients under ipilimumab the incidence of secondary hypothyroidism is similar to the incidence of hypophysitis (0-17\%) (112). Notably, in the majority of cases, endocrine immune-related adverse events are irreversible and lifelong replacement therapy is required.

In two studies, tremelimumab was associated with thyroid dysfunction in $4 \%$ of patients $(113,114)$. The incidence of ipilimumab-induced primary thyroid dysfunction appeared lower (0-2\%) when ipilimumab was administered at standard dose $(3 \mathrm{mg} / \mathrm{kg})$, as a single agent or in combination with $\mathrm{CT}$. In small reports, higher dosage of ipilimumab alone 


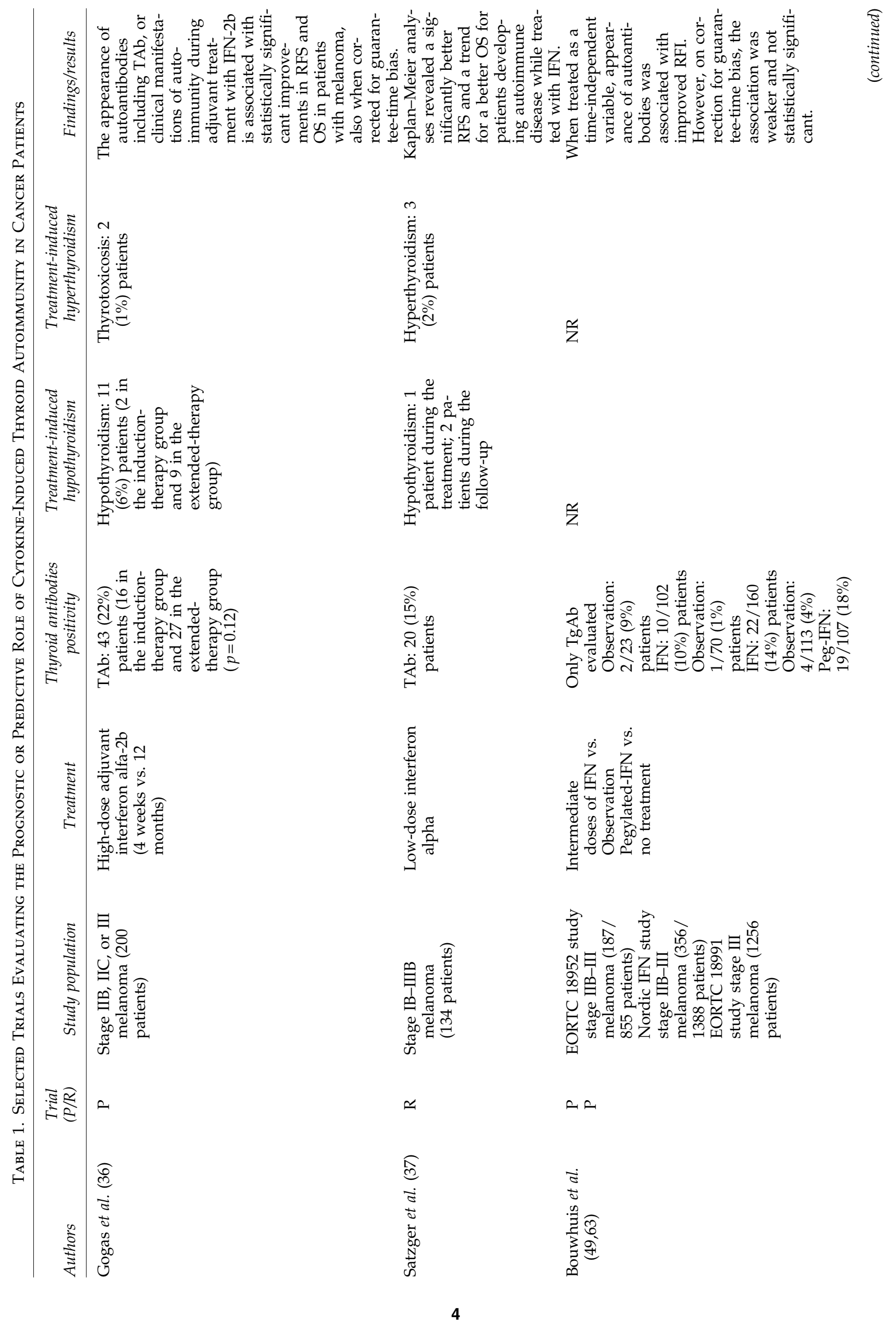




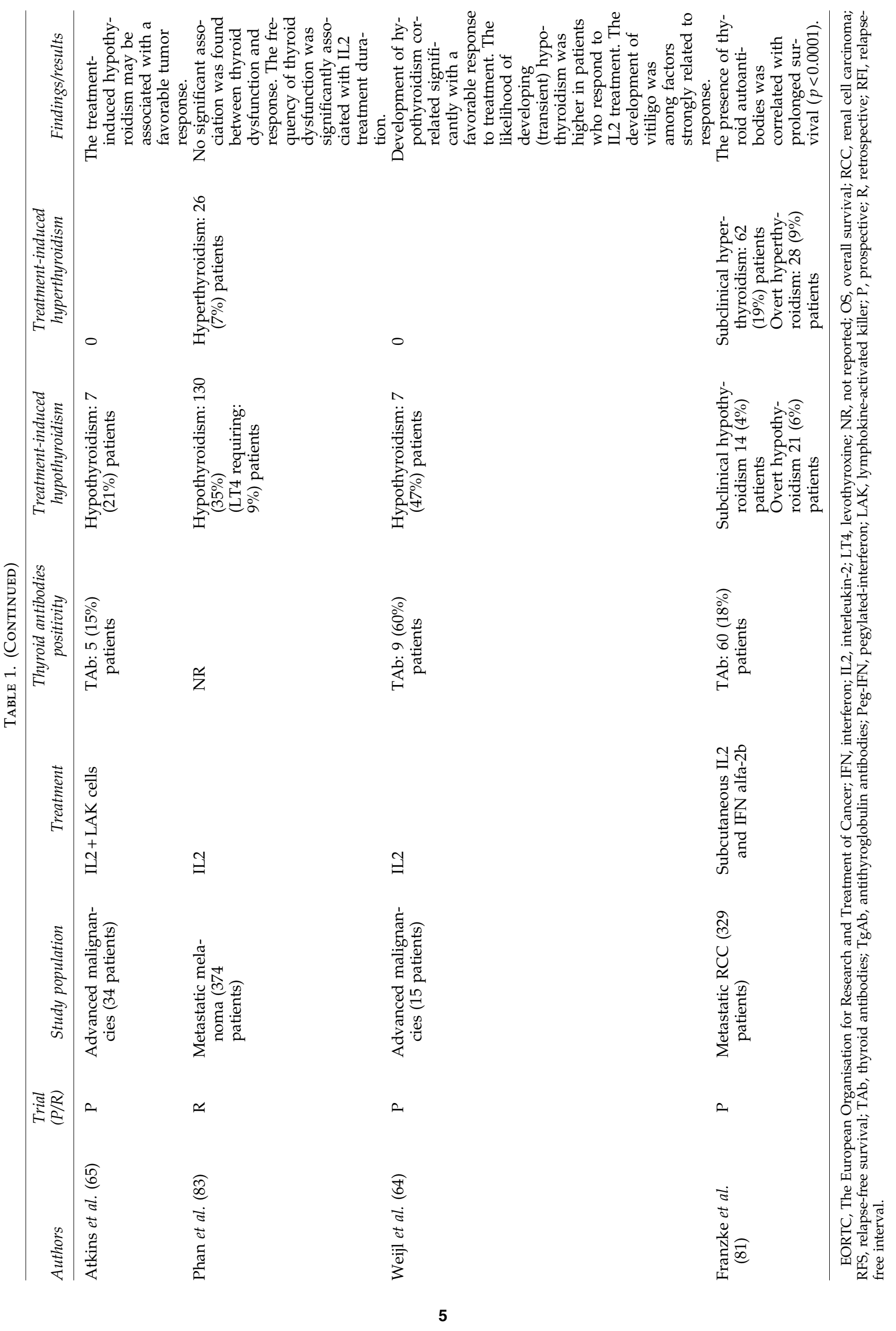


(10 mg/ $\mathrm{kg}$ ) or in combination with bevacizumab $(7.5-15 \mathrm{mg} /$ $\mathrm{kg}$ ), an anti-VEGF agent, was associated with a higher rate of thyroiditis (7\% and 19\% of cases, respectively) $(115,116)$. In patients with metastatic prostate cancer receiving ipilimumab at increasing doses in combination with an anti-prostate specific antigen vaccine, hypothyroidism was diagnosed in 4 (13.3\%) patients at the higher dose levels (5-10 mg/kg) (117). Conversely, no cases of endocrinopathy were reported in 36 patients receiving ipilimumab $(0.1-3 \mathrm{mg} / \mathrm{kg})$ combined with IL2 (118). The anti-CTLA4-mAb thyroid damage presents as thyroiditis associated with $\mathrm{TAb}$ and hypothyroidism, or transient hyperthyroidism. Rare cases of Graves' ophthalmopathy have also been reported, with elevation of TSH-receptor antibodies but normal thyroid function $(119,120)$. However, clinical details are still scarce. Similar to antiCTLA4-MAb hypophysitis, the onset of anti-CTLA4-MAb thyreopathy occurs after 2-4 infusions. Most cases showed subclinical course or may be transient, consistent with a silent autoimmune thyroiditis. Alternatively, it may evolve into permanent hypothyroidism, requiring thyroid hormone supplementation (106). It is unknown whether the administration of anti-CTLA4-MAbs may worsen previous thyroiditis, as patients with previous autoimmune disease were not included in clinical trials evaluating these agents.

MAb blocking PD-1 or one of its ligands (PD-1L) are at an early phase of clinical development $(103,121)$. Currently, these agents seemed to only slightly affect the endocrine system. Hypothyroidism was reported in $2-3 \%$ of patients receiving higher doses of these drugs $(122,123)$. Further studies are needed to better define the pathogenic mechanism of primary and secondary thyroid dysfunction induced by immune checkpoint inhibitors.

\section{Alemtuzumab}

Alemtuzumab is a humanized MAb binding the CD52 cell surface antigen on lymphocytes and monocytes. The drug induces complement-mediated lysis of these cells exiting in intense lymphopenia (124). It is approved for high-risk or pretreated B-cell chronic lymphocytic leukemia (125). Alemtuzumab is also used as an immune suppressor in several conditions, including stem cell transplants, graft-versus-host disease after allogeneic stem cell transplant, and multiple sclerosis (MS) (126-128). Thyroid dysfunction has been reported with alemtuzumab in patients who received the drug for autoimmune disease $(124,129,130)$, but not for cancer or rheumatoid arthritis $(131,132)$. In a large study, thyroid dysfunction was found in 48/216 (22\%) patients with MS treated with alemtuzumab (126). Autoimmune hypothyroidism was reported in $6.9 \%$ and thyroiditis in $4.2 \%$, but Graves' disease in $14.8 \%$ of patients. In another study, $30 \%$ of MS patients developed Graves' disease, 9-31 months after a course of alemtuzumab (133). Notably, the alemtuzumab-induced autoimmune effects are mostly antibody mediated (type-2 hypersensitivity), including autoimmune neutropenia, thrombocytopenic purpura, and Goodpasture syndrome (134). The reasons underlying the lack of thyroid dysfunction in cancer patients compared to patients with MS are unknown.

\section{lodine-Based Anticancer Radioimmunotherapy}

In cancer radioimmunotherapy, an antibody with specificity for a tumor-associated antigen is used to deliver a lethal dose of radiation to the tumor cells during isotopic decay (135). Tositumomab, an anti-CD20 MAb combined with ${ }^{131} \mathrm{I}$, is approved for the treatment of non-Hodgkin lymphoma, whereas ${ }^{131}$ I-metaiodobenzylguanidine is used in pheochromocytoma, neuroblastoma and carcinoid tumors. As the radioactive iodine concentrates in thyroid cells, hypothyroidism may occur in 9-64\% of patients (within 6-24 months or later) (136-141). However, this side effect is preventable by the administration of oral iodine (Lugol solution or saturated solution of potassium iodide, SSKI) or potassium perchlorate $\left(\mathrm{KClO}_{4}\right)$.

\section{Tyrosine Kinase Inhibitors}

Tyrosine kinase inhibitors (TKI) are small molecules exerting their therapeutic activity by interfering with kinasereceptors that are critical for tumor cell growth, invasion, metastasis, and angiogenesis. Currently, several TKI are approved as anticancer therapeutics and approximately 150 kinase-targeted drugs are under clinical evaluation (142). Despite their selectivity, TKI show variable affinity for different kinase-receptors and none is specific for a single kinasereceptor. Therefore, off-target intracellular activities of normal cells are frequently impaired, leading to a variety of toxicities, often unusual, such as those to the endocrine system. TKIinduced endocrine side effects mainly include thyroid dysfunction, whereas gonadal and adrenal functions, bone, and glucose metabolism are less frequently or occasionally affected (143). TKI may cause de novo hypothyroidism or hyperthyroidism, or worsen pre-existing hypothyroidism, thus increasing thyroid hormone requirements in patients on levothyroxine (LT4) replacement. When hyperthyroidism occurs, it may represent the transient thyrotoxicosis phase of a destructive thyroiditis, often followed by hypothyroidism (144). These thyroid dysfunctions may be induced by TKI that inhibit key kinase-receptors in angiogenic pathways, but not by TKI targeting other kinase-receptors, such as epidermal growth factor receptors family (Table 1).

In cancer patients with normal thyroid function, TKI may cause abnormalities in thyroid laboratory tests, primary hypothyroidism (overt hypothyroidism in 32-85\%, subclinical hypothyroidism in up to $100 \%$ ), or decrease in TSH levels/ thyrotoxicosis (transient in $0-24 \%$, persistent in $0-5 \%$ of cases) (145) (Table 2). The onset of TKI-induced thyroid dysfunction is largely variable, ranging from 4 to 94 weeks after initiation of the treatment (median 4 weeks in prospective studies) (146). Whether prolonged treatment with a TKI, or previous treatments with cytokines (i.e., IFN/IL-2) or another TKI might influence the incidence or the course of thyroid dysfunction remains to be clarified. Among TKI approved for clinical use, sunitinib, a TKI mainly targeting angiogenic kinase-receptors, exposes patients to a higher risk of developing TKI hypothyroidism (14-71\% of patients in prospective studies) (146-153). Thyroid dysfunction has also been described with other antiangiogenic TKI, such as sorafenib, motesanib, pazopanib, cediranib, and linifarib, but at lower rates (154-178) (Table 2). Nilotinib and dasatinib are secondgeneration TKI approved for the treatment of Philadelphiapositive chronic myeloid leukemia, showing structural similarity to imatinib, but greater potency and specificity for inhibition of BCR-ABL $(179,180)$. Thyroid abnormalities during treatment with imatinib, nilotinib, and dasatinib were 
retrospectively reported in $25 \%, 55 \%$, and $70 \%$, respectively (181). In patients under nilotinib, hypothyroidism was diagnosed in 12/55 (22\%) patients, (6 subclinical, 6 clinical) and hyperthyroidism in another $18(33 \%)$ patients (10 subclinical, 8 clinical), while $4 / 55$ (7\%) patients had evidence of thyroid autoimmunity (181). Similar results were reported with dasatanib (181) (Table 2). Compared with imatinib, nilotinib and dasatanib rarely altered the requirement of LT4 replacement therapy. The causes of the above difference are still unexplained. However, thyroid toxicity induced by newer TKI need to be better evaluated in prospective clinical trials.

Several mechanisms have been suggested to explain the onset of thyroid dysfunction in patients under TKI treatment. As thyrotoxicosis in a few cases preceded the development of hypothyroidism and thyroid atrophy, destructive thyroiditis might have triggered both adverse events $(144,147)$. Other possible mechanisms include direct toxic effects on thyrocytes, leading to an impaired iodine uptake (148) and a reduced synthesis of thyroid hormones related to inhibition of TPO activity (149). However, altered iodine uptake has not been confirmed by in vitro studies (182). Similarly, a direct effect against TPO induced by TKI seems an improbable explanation in cases with initial destructive thyrotoxicosis or with thyroid atrophy. Induction of Hashimoto's thyroiditis has also been proposed as another potential mechanism (183). However, TAb infrequently increased during TKI treatment (146).

More likely, the TKI-induced thyroid dysfunction may derive from the inhibition exerted by these agents on kinasereceptors of angiogenic pathways, such as VEGF receptors 1-3 and platelet-derived growth factor receptor (PDGFR). TKI-induced regression of thyroid vascular bed with significant capillary alteration and reduction in density has been demonstrated in experimental animal models $(184,185)$. This could cause the reduction of blood flow in the thyroid, an extremely vascular gland. If the thyroid blood flow decreases rapidly, an ischemic thyroiditis could result, leading to transient thyrotoxicosis (186). If the decreased blood flow develops more slowly, gradual thyroid destruction may occur, resulting in hypothyroidism (186). In humans, reduced thyroid volume and vascularization by Doppler ultrasound rapidly recovered after cessation of sunitinib $(186,187)$. The reduced thyroid volume induced by reduction in blood flow may also explain the impaired iodine uptake seen in vivo but not in vitro (188). Notably, targeting different molecular components of angiogenesis (receptors vs. ligand) does not trigger the same toxic effect. Unlike antiangiogenic TKI inhibiting vascular endothelial growth factor receptor (VEGFR)/PDGFR, bevacizumab (which targets VEGF-A) occasionally altered thyroid homeostasis $(189,190)$.

The reason why some TKI do not affect thyroid or do this at a lesser extent is unclear. In comparative studies, sunitinib was shown to induce thyroid damage more frequently than other TKI (i.e., sorafenib). This might be because sunitinib inhibits more than a single kinase-receptor regulating normal and tumor angiogenesis (not only VEGFR2, but also PDGFR and VEGFR1) and more potently than other TKI. The thyroid gland shows the highest blood flow rates per unit weight of any tissue in the body (191). Physiologically, thyroid angiogenesis is regulated mainly by VEGF signaling, but under ischemia/hypoxia PDGF/PDGFR are upregulated to exert a compensatory function $(192,193)$.
The entire sunitinib-induced inhibition of both angiogenic key molecules (i.e., VEGFR2) and upregulated compensatory receptors (i.e., PDGFR) may result more frequently in thyroid ischemia via capillary regression and constriction (194). This, in turn, might trigger both destructive thyroiditis and thyroid dysfunction (194). This hypothesis might also explain the rarity of thyroid dysfunction induced by bevacizumab, which binds VEGF-A, but not VEGFRs and PDGFR.

The worsening of appropriately treated hypothyroidism in thyroidectomized patients under imatinib, a TKI mainly targeting c-KIT, was the first thyroid hormone abnormality described with TKI (195). Imatinib does not alter euthyroidism in nonthyroidectomized patients (196). An interference with the nondeiodination clearance of LT4 was initially thought to explain the increased LT4 requirement induced by imatinib (195). Several drugs (i.e., phenobarbital, phenytoin, carbamazepine, rifampicin, and nicardipine) increase thyroid hormone clearance and worsen hypothyroidism in patients under LT4, by inducing hepatic microsomal enzymes $(12,197-$ 200), and imatinib is a potent competitive inhibitor of mixed function oxygenases (CYP2C9, CYP2D6, CYP3A4/5) (195). Consistently, TSH levels return to normal after discontinuation of imatinib (195).

However, recent studies showed that other TKI may increase the LT4 requirement in thyroidectomized hypothyroid patients by interfering at different steps of thyroid hormone metabolism (171,201-205) (Table 2). In thyroidectomized patients on sorafenib, decreased T3/T4 and T3/rT3 ratios, accompanied by doubling in TSH levels, were found (202). In rats, sunitinib induced a decrease in serum T4 and T3 levels, increased deiodinase-3 (DIO3), and decreased deiodinase-1 activity, together with marked thyroid capillary regression (206). Also, it was suggested that motesanib and vandetanib might increase LT4 metabolism via an increased activity of DIO3 in peripheral tissue $(171,204,205)$ in thyroidectomized patients, while hypothyroidism is infrequently reported in patients with thyroid in situ (172). Interestingly, in patients treated with sunitinib, not only did the T3/reverse-T3 ratio decrease and the TSH levels show a twofold increase, but following sunitinib withdrawal, DIO3 activity reversed, whereas thyroid hormone levels remained low (206). Further evidence suggests an impairment in deiodases activity in TKIinduced hypothyroidism. Indeed, in this condition, TSH levels are often inappropriately high for the concomitant serum free T3 and free T4 levels $(194,207)$. This might be related to a far more reduced activity of DIO2 (expressed in the pituitary gland) than of deiodinase-1 (expressed mainly in the liver and kidney), leading to an intracellular depletion of T3 in the thyrotrophs and an inappropriately high TSH level (194). Additionally, in a new mouse model lacking DIO2 activity in pituitary thyrotrophs, normal serum T3 and high serum TSH and T4 levels were found, despite an euthyroid status (208).

Moreover, TKI may interfere with thyroid hormones even at central level, by inhibiting the monocarboxylate transporter-8, a thyroid hormone trans-membrane transporter expressed in the brain (including the hypothalamus and pituitary) and other tissues (i.e., liver, kidney, thyroid). In an in vitro model, several TKIs, including sunitinib and imatinib, dose dependently and noncompetitively inhibit T4/T3 uptake, inducing cellular depletion of T3 (209). However, whether this mechanism is relevant in humans requires further investigation. 
Table 2. Overview of Results from Clinical Trials Evaluating Thyroid Dysfunction Induced by TyRosine Kinase INHIBITORS

\begin{tabular}{|c|c|c|c|c|c|c|}
\hline Drug & Targeted kinases & $\begin{array}{c}\text { Approval/clinical } \\
\text { development }\end{array}$ & Authors & Type of study & Cancer type & $\begin{array}{l}\text { No. of } \\
\text { patients }\end{array}$ \\
\hline \multirow[t]{9}{*}{ Sunitinib } & \multirow{9}{*}{$\begin{array}{l}\text { VEGFRs-1-3, } \\
\text { PDGFR- } \beta \text {; C-KIT; } \\
\text { FLT-3 }\end{array}$} & \multirow{9}{*}{$\begin{array}{l}\text { Approved for patients } \\
\text { with RCC or GIST. } \\
\text { Under evaluation for } \\
\text { several tumor types, } \\
\text { including breast, } \\
\text { lung, thyroid, and } \\
\text { colorectal cancer. }\end{array}$} & Desai et al. (147) & $\mathrm{P}$ & GIST & 42 \\
\hline & & & Mannavola et al. (148) & $\mathrm{P}$ & GIST & 24 \\
\hline & & & Wong et al. (149) & $\mathrm{R}$ & $\begin{array}{l}\text { Solid } \\
\text { tumors }\end{array}$ & 40 \\
\hline & & & Rini et al. (185) & $\mathrm{R} / \mathrm{P}$ & $\mathrm{RCC}$ & 66 \\
\hline & & & Wolter et al. (146) & $\mathrm{P}$ & RCC/GIST & 59 \\
\hline & & & Shinohara et al. (150) & $\mathrm{P}$ & $\mathrm{RCC}$ & $17^{\mathrm{c}}$ \\
\hline & & & Baldazzi et al. (151) & $\mathrm{P}$ & $\mathrm{RCC}$ & 22 \\
\hline & & & Rutkowski et al. (152) & $\mathrm{P}$ & GIST & 137 \\
\hline & & & Sabatier et al. (153) & $\mathrm{P}$ & RCC & 111 \\
\hline \multirow[t]{5}{*}{ Sorafenib } & \multirow{5}{*}{$\begin{array}{l}\text { VEGFRs, } \\
\text { PDGFR- } \beta \text {, } \\
\text { c-KIT, RET, } \\
\text { B-RAF }\end{array}$} & \multirow{5}{*}{$\begin{array}{l}\text { Approved for patients } \\
\text { with RCC and HCC. } \\
\text { Evaluated in lung, } \\
\text { pancreatic, prostate, } \\
\text { melanoma and DTC. }\end{array}$} & Tamaskar et al. (154) & $\mathrm{P}$ & RCC & 39 \\
\hline & & & Clement et al. (155) & $\mathrm{P}$ & RCC & 23 \\
\hline & & & Miyake et al. (156) & $\mathrm{P}$ & $\mathrm{RCC}$ & 69 \\
\hline & & & Abdulrahman et al. (202) & $\mathrm{P}$ & MTC & $21^{\mathrm{d}}$ \\
\hline & & & Di Lorenzo et al. (157) & $\mathrm{P}$ & RCC & 52 \\
\hline $\begin{array}{l}\text { Sunitinib/ } \\
\text { Sorafenib }\end{array}$ & See above & See above & Riesenbeck et al. (158) & $\mathrm{P}$ & RCC & $\begin{array}{l}52 \\
31\end{array}$ \\
\hline $\begin{array}{l}\text { Sunitinib/ } \\
\text { Sorafenib }\end{array}$ & See above & See above & Schmidinger et al. (159) & $\mathrm{P}$ & RCC & $\begin{array}{l}41 \\
37\end{array}$ \\
\hline $\begin{array}{l}\text { Sunitinib/ } \\
\text { Sorafenib }\end{array}$ & See above & See above & Kitajima et al. (160) & $\mathrm{P}$ & $\mathrm{RCC}$ & $\begin{array}{l}17 \\
25\end{array}$ \\
\hline $\begin{array}{l}\text { Sunitinib/ } \\
\text { Sorafenib }\end{array}$ & See above & See above & Clemons et al. (161) & $\mathrm{R}$ & $\mathrm{RCC}$ & $\begin{array}{l}34 \\
22\end{array}$ \\
\hline $\begin{array}{l}\text { Sunitinib/ } \\
\text { Sorafenib }\end{array}$ & See above & See above & Feldt et al. (162) & $\begin{array}{c}\mathrm{R} \\
\text { (postmarket) }\end{array}$ & $\mathrm{RCC}$ & $\begin{array}{l}1295 \\
1214\end{array}$ \\
\hline $\begin{array}{l}\text { Sunitinib/ } \\
\text { Sorafenib }\end{array}$ & See above & See above & Zhao et al. (163) & $\mathrm{R}$ & $\begin{array}{l}\text { Early } \\
\text { RCC }\end{array}$ & $\begin{array}{l}23 \\
20\end{array}$ \\
\hline
\end{tabular}




\begin{tabular}{|c|c|c|c|c|c|c|c|}
\hline $\begin{array}{c}\text { No. of patients } \\
\text { with altered } \\
\text { TFTs after } \\
\text { TKI } \\
\text { treatment } \\
(\%)\end{array}$ & $\begin{array}{c}\text { No. of } \\
\text { patients with } \\
\text { positive } \\
\text { TAb/evaluated } \\
(\%)\end{array}$ & $\begin{array}{c}\text { No. of patients } \\
\text { with increased } \\
\text { TSH after } \\
\text { TKI treatment } \\
(\%)\end{array}$ & $\begin{array}{l}\text { Overt hypo } \\
\text { thyroidism } \\
\text { after TKI } \\
\text { treatment }(\%)\end{array}$ & $\begin{array}{c}\text { No. of patients } \\
\text { with decreased } \\
\text { TSH after } \\
\text { TKI treatment (\%) }\end{array}$ & $\begin{array}{l}\text { Time to onset } \\
\text { of thyroid } \\
\text { dysfunction } \\
\text { (median) }\end{array}$ & $\begin{array}{c}\text { No. of } \\
\text { patients } \\
\text { who needed } \\
\text { an increased } \\
\text { LT4 dosage } \\
(\%)\end{array}$ & $\begin{array}{c}\text { Prognostic } \\
\text { value of } \\
\text { TKI-induced } \\
\text { hypothyroidism }\end{array}$ \\
\hline $26 / 42(62)$ & $0 / 2$ & $22 / 42(52)$ & $15 / 42(36)$ & $4 / 42(10)$ & $\begin{array}{c}50(12-94) \\
\text { weeks }\end{array}$ & NR & NR \\
\hline $16 / 24(71)$ & $0 / 23$ & $16 / 24(71)$ & $10 / 24(46)$ & $0 / 24$ & NR & NR & NR \\
\hline $24 / 40(60)$ & NR & $21 / 40(53)$ & $14 / 40(35)$ & $3 / 40(8)$ & $\begin{array}{l}5 \text { (1-36) } \\
\text { months }\end{array}$ & $4 / 40(10)$ & NR \\
\hline $56 / 66(85)$ & $\begin{array}{c}13 / 44(30) \\
(\mathrm{TgAb})\end{array}$ & $46 / 66(70)$ & $17 / 66(26)^{b}$ & $0 / 66$ & $\begin{array}{c}2(1-14) \\
\text { cycles }\end{array}$ & NR & Uncertain \\
\hline $25 / 59(42)$ & $2 / 55(4)$ & $\begin{array}{l}20 / 59(34) \\
\text { transient }\end{array}$ & $16 / 59(27)$ & $3 / 59(5)$ & $\begin{array}{l}4(2-46) \\
\text { weeks }\end{array}$ & NR & NR \\
\hline 9/17 (53) & $0 / 6$ & 1/17 (6) & $8 / 17(53)$ & $\begin{array}{l}\text { TSH suppressed } \\
\text { in } 4 / 8 \text { patients } \\
\text { (transiently) }\end{array}$ & NR & NR & NR \\
\hline $13 / 22(59)$ & $0 / 22$ & $13 / 22(59)$ & $2 / 22(9)$ & $0 / 22$ & $\begin{array}{r}3.3(1-7) \\
\text { cycles }\end{array}$ & NA & Yes \\
\hline NR & NR & NR & 42/137 (31) & 0/137 & NR & NR & No \\
\hline $54 / 102(53)$ & NR & $51 / 102(50)$ & $46 / 102(45)$ & $3 / 102(3)$ & $\begin{array}{l}5.4(0.6-22) \\
\text { months }\end{array}$ & NR & No \\
\hline $16 / 43(43)$ & 2/7 (29) & NR & 7/39 (18) & $\begin{array}{l}1 / 39(3) \\
\quad \text { transient }\end{array}$ & $\begin{array}{c}1.8(0.6-7.3) \\
\text { months }\end{array}$ & NR & \\
\hline $15 / 23(65)$ & NR & $7 / 23(30)$ & NR & NR & NR & NR & NR \\
\hline $46 / 69(68)$ & NR & $46 / 69(68)$ & $4 / 69(6)$ & $\begin{array}{l}11 / 46(24) \\
\text { transient }\end{array}$ & $\begin{array}{c}1.7(0.7-7.5) \\
\text { months }\end{array}$ & NR & NR \\
\hline $21 / 21(100)$ & NR & $21 / 21(100)$ & NA & NA & NA & 100 & NA \\
\hline NR & NR & NR & 4/52 (8) & $0 / 52$ & NR & NR & NR \\
\hline $\begin{array}{l}13 / 52(25) \\
8 / 31(26)\end{array}$ & NR & NR & $\begin{array}{l}13 / 21(62) \\
8 / 21(38)\end{array}$ & NR & $\begin{array}{l}\text { 16/21 }(76 \%) \\
\text { patients } \\
\text { showed } \\
\text { TSH } \\
\text { increase } \\
\text { within } \\
\text { the first } 4 \\
\text { weeks } \\
\text { of treat- } \\
\text { ment }\end{array}$ & NR & Yes \\
\hline NR & NR & $\begin{array}{l}12 / 41(29) \\
18 / 37(49)\end{array}$ & NR & NR & $\begin{array}{l}\text { 25/78 }(32 \%) \\
\text { patients } \\
\text { showed } \\
\text { TSH in- } \\
\text { crease } \\
\text { within the } \\
\text { first } \\
4 \text { weeks of } \\
\text { treatment }\end{array}$ & $7 / 14(50)$ & Yes \\
\hline NR & NR & NR & $\begin{array}{l}12 / 17(71) \\
9 / 25(36)\end{array}$ & NR & $\begin{array}{l}95 \text { (12-315) } \\
\text { days }\end{array}$ & NR & NR \\
\hline $\begin{array}{l}\text { NR } \\
\text { NR }\end{array}$ & $\begin{array}{l}\text { NR } \\
\text { NR }\end{array}$ & $\begin{array}{c}\text { 12/34 (35) } \\
\text { persist } \\
3 / 34(9) \\
\text { transient } \\
3 / 22(14) \\
\text { transient } \\
3 / 22(14) \\
\text { persist }\end{array}$ & $\begin{array}{l}15 / 34(44) \\
6 / 22(27)\end{array}$ & 0 & $\begin{array}{l}11 \text { months } \\
\text { (range NR) } \\
20 \text { months } \\
\text { (range NR) }\end{array}$ & $\begin{array}{l}8 / 11(73) \\
2 / 6(33)\end{array}$ & NR \\
\hline NR & NR & $\begin{array}{c}178 / 1295(14) \\
77 / 1214(6)\end{array}$ & $\begin{array}{c}178 / 1295(14) \\
77 / 1214(6)\end{array}$ & $\begin{array}{l}35 / 1295 \text { (3) persist } \\
42 / 1214 \text { (4) persist }\end{array}$ & NR & NR & NR \\
\hline NR & NR & NR & $\begin{array}{l}9 / 23(39) \\
3 / 20(15)\end{array}$ & NR & NR & NR & NR \\
\hline
\end{tabular}


TABLe 2. (CONTinued)

\begin{tabular}{|c|c|c|c|c|c|c|}
\hline Drug & Targeted kinases & $\begin{array}{l}\text { Approval/clinical } \\
\text { development }\end{array}$ & Authors & Type of study & Cancer type & $\begin{array}{l}\text { No. of } \\
\text { patients }^{\mathrm{a}}\end{array}$ \\
\hline \multirow[t]{4}{*}{ Axitinib } & \multirow[t]{4}{*}{ VEGFRs 1-3 } & \multirow{4}{*}{$\begin{array}{l}\text { Approved for use in } \\
\text { patients with RCC } \\
\text { that failed to respond } \\
\text { to a previous } \\
\text { treatment. }\end{array}$} & Fujiwara et al. (165) & $\mathrm{P}$ (phase I) & $\begin{array}{l}\text { Solid } \\
\text { tumors }\end{array}$ & $18^{\mathrm{c}}$ \\
\hline & & & Mukohara et al. (164) & $\mathrm{P}$ & $\begin{array}{l}\text { Solid } \\
\text { tumors }\end{array}$ & 12 \\
\hline & & & Tomita et al. (166) & $\mathrm{P}$ & RCC & $64^{c}$ \\
\hline & & & Rini et al. (167) & $\mathrm{P}$ & $\mathrm{RCC}$ & 62 \\
\hline $\begin{array}{l}\text { Axitinib/ } \\
\text { Sorafenib }\end{array}$ & See above & See above & Rini et al. (168) & P (phase III) & $\mathrm{RCC}$ & $\begin{array}{l}359 \\
355\end{array}$ \\
\hline $\begin{array}{l}\text { Sunitinib/ } \\
\text { Sorafenib/ } \\
\text { Axitinib }\end{array}$ & See above & See above & Daimon et al. (169) & $\mathrm{R}$ & RCC & $\begin{array}{c}15 \\
12 \\
6\end{array}$ \\
\hline Vatalanib & $\begin{array}{l}\text { VEGFRs (VEGFR2), } \\
\text { PDGFR, KIT }\end{array}$ & $\begin{array}{l}\text { Evaluated in various } \\
\text { solid and hemaologi- } \\
\text { cal cancers }\end{array}$ & Joensuu (170) & $\mathrm{P}$ & GIST & 45 \\
\hline Vandetanib & $\begin{array}{l}\text { VEGFR-2, EGFR, } \\
\text { RET }\end{array}$ & $\begin{array}{l}\text { MTC (advanced or } \\
\text { unresectable) }\end{array}$ & Robinson et al. (205) & $\mathrm{P}$ & MTC & $17^{\mathrm{d}}$ \\
\hline \multirow[t]{3}{*}{ Motesanib } & \multirow{3}{*}{$\begin{array}{l}\text { VEGFRs } 1-3, \\
\text { PDGFR- } \beta \text {, } \\
\text { KIT, and RET }\end{array}$} & \multirow{3}{*}{$\begin{array}{l}\text { Under clinical evalua- } \\
\text { tion in various } \\
\text { cancers }\end{array}$} & Sherman et al. (171) & $\mathrm{P}$ & DTC & $93^{\mathrm{d}}$ \\
\hline & & & Schlumberger et al. (204) & $\mathrm{P}$ & MTC & $91^{\mathrm{d}}$ \\
\hline & & & Blumenschein et al. (172) & $\mathrm{P}$ & $\begin{array}{l}\text { NSCLC } \\
\text { Motesanib }\end{array}$ & 181 \\
\hline Pazopanib & $\begin{array}{l}\text { VEGFRs, } \\
\text { PDGFR, KIT }\end{array}$ & $\begin{array}{l}\text { Approved for use in } \\
\text { RCC and soft tissue } \\
\text { sarcomas. Active in } \\
\text { OC } \\
\text { and NSCLC. }\end{array}$ & Sternberg et al. (173) & $\mathrm{P}$ & $\begin{array}{l}\text { Bev } 15 \mathrm{mg} / \mathrm{Kg} \\
\text { RCC }\end{array}$ & 290 \\
\hline \multirow[t]{2}{*}{ Cediranib } & \multirow[t]{2}{*}{ VEGFRs $1-3$, } & \multirow{2}{*}{$\begin{array}{l}\text { Under clinical } \\
\text { evaluation in RCC, } \\
\text { NSCLC, CNS cancers }\end{array}$} & Matulonis et al. (174) & $\mathrm{P}$ & Ovary & 46 \\
\hline & & & Garland et al. (175) & $\mathrm{P}$ & PM & 47 \\
\hline \multirow[t]{3}{*}{ Linifanib } & \multirow{3}{*}{$\begin{array}{l}\text { VEGFR-1,-2, } \\
\text { PDGFR- } \beta \text {, CSFR- } \\
1, \text { fms-3 }\end{array}$} & \multirow{3}{*}{$\begin{array}{l}\text { Under clinical evalua- } \\
\text { tion in NSCLC; HCC; } \\
\text { RCC }\end{array}$} & Asahina et al. (176) & $\mathrm{P}$ (phase I) & Solid tumors & 18 \\
\hline & & & Toh et al. (177) & $\mathrm{P}$ & $\mathrm{HCC}$ & 44 \\
\hline & & & Tan et al. (178) & $\mathrm{P}$ & NSCLC & 139 \\
\hline Imatinib & $\begin{array}{l}\text { BCR-ABL, c-Kit, } \\
\text { PDGFR- } \beta, \text { RET, } \\
\text { c-Fms }\end{array}$ & $\begin{array}{l}\text { Approved for use } \\
\text { in CML and GISTs }\end{array}$ & de Groot et al. (195) & $\mathrm{R}$ & GIST/MTC & 11 \\
\hline Imatinib & See above & See above & Kim et al. (181) & $\mathrm{R}$ & CML & 8 \\
\hline Nilotinib & $\begin{array}{l}\text { BCR-ABL, c-KIT, } \\
\text { PDGFR- } \beta\end{array}$ & $\begin{array}{l}\text { Approved for use } \\
\text { in drug-resistant } \\
\text { CML }\end{array}$ & & $\mathrm{R}$ & CML & 55 \\
\hline Dasatanib & $\begin{array}{l}\text { BCR/ABL, } \\
\text { Src, c-Kit }\end{array}$ & $\begin{array}{l}\text { Approved as } 1^{\text {st }} \text { - line } \\
\text { therapy in CML } \\
\text { and } \mathrm{Ph}+\mathrm{ALL} \text {; under } \\
\text { evaluation in several } \\
\text { cancers types. }\end{array}$ & & $\mathrm{R}$ & CML & 10 \\
\hline
\end{tabular}

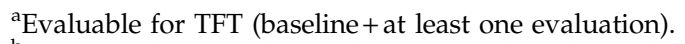

${ }^{\mathrm{b}}$ Patients who started LT4 due to overt hypothyroidism.

'Only Japanese patients included.

${ }^{\mathrm{d}}$ All patients were under LT4 replacement therapy due to thyroidectomy.

Bev, Bevacizumab; CML, chronic myelogenous leukemia; CSFR-1, colony-stimulating factor 1 receptor; DTC, differentiated thyroid cancer; GIST, gastrointestinal stromal tumor; HCC, hepatocellular carcinoma; MTC, medullary thyroid cancer; NA, not applicable; NSCLC, nonsmall cell lung cancer; PDGFR, platelet derived growth factor receptor; Ph + ALL, Philadelphia chromosome-positive acute lymphoblastic leukemia; PM, pleural mesothelioma; RCC, renal cell carcinoma; TFTs, thyroid function tests; TKI, tyrosine kinase inhibitor; TSH, thyrotropin; VEGFRs, vascular endothelial growth factor receptors. c-KIT, v-kit Hardy-Zuckerman 4 feline sarcoma viral oncogene homolog; FLT3, Fms (feline McDonough sarcoma virus) -like tyrosine kinase 3; RET, rearranged during transfection; B-RAF, v-Raf (rapidly accelerated fibrosarcoma) murine sarcoma viral oncogene homolog B1; OC, ovarian cancer; CNS, central nervous system; BCR-ABL: breakpoint cluster region-Abelson gene. 


\begin{tabular}{|c|c|c|c|c|c|c|c|}
\hline $\begin{array}{l}\text { No. of patients } \\
\text { with altered } \\
\text { TFTs after } \\
\text { TKI } \\
\text { treatment } \\
(\%)\end{array}$ & $\begin{array}{c}\text { No. of } \\
\text { patients with } \\
\text { positive } \\
\text { TAb/evaluated } \\
(\%)\end{array}$ & $\begin{array}{c}\text { No. of patients } \\
\text { with increased } \\
\text { TSH after } \\
\text { TKI treatment } \\
(\%)\end{array}$ & $\begin{array}{l}\text { Overt hypo } \\
\text { thyroidism } \\
\text { after TKI } \\
\text { treatment }(\%)\end{array}$ & $\begin{array}{c}\text { No. of patients } \\
\text { with decreased } \\
\text { TSH after } \\
\text { TKI treatment (\%) }\end{array}$ & $\begin{array}{l}\text { Time to onset } \\
\text { of thyroid } \\
\text { dysfunction } \\
\text { (median) }\end{array}$ & $\begin{array}{c}\text { No. of } \\
\text { patients } \\
\text { who needed } \\
\text { an increased } \\
\text { LT4 dosage } \\
(\%)\end{array}$ & $\begin{array}{c}\text { Prognostic } \\
\text { value of } \\
\text { TKI-induced } \\
\text { hypothyroidism }\end{array}$ \\
\hline 16/18 (89) & NR & 16/18 (89) & NR & $0 / 18$ & NR & NR & NR \\
\hline 11/12 (92) & NR & 11/12 (92) & $7 / 12(58)$ & $\begin{array}{l}3 / 12(25) \\
\text { transient }\end{array}$ & $\begin{array}{l}\text { Within } 1 \\
\text { month } \\
\text { (range NR) }\end{array}$ & NR & NR \\
\hline $56 / 68(88)$ & NR & NR & $31 / 64(48)$ & NR & NR & NR & NR \\
\hline NR & NR & NR & $11 / 62(18)$ & $0 / 62$ & NR & NR & NR \\
\hline NR & NR & NR & $\begin{array}{l}69 / 359(19) \\
29 / 355(8)\end{array}$ & NR & $\begin{array}{l}\text { NR } \\
\text { NR }\end{array}$ & NR & Yes \\
\hline $\begin{array}{l}9 / 15(60) \\
6 / 12(50)\end{array}$ & $\begin{array}{l}4(27) \\
1(8)\end{array}$ & NR & $\begin{array}{l}7 / 15(47) \\
3 / 12(25)\end{array}$ & $\begin{array}{c}0 / 4(27) \\
0\end{array}$ & $\begin{array}{l}16 \text { (range } \\
\text { NR) weeks }\end{array}$ & NR & NR \\
\hline 6/6 (100) & 0 & & 6/6 (100) & 0 & $\begin{array}{l}16 \text { (range } \\
\text { NR) weeks } \\
3 \text { (range NR) } \\
\text { weeks }\end{array}$ & & \\
\hline $1 / 45(2)$ & NR & NR & $1 / 45(2)$ & NR & NR & NR & NR \\
\hline NR & NR & $17 / 17(100)$ & NR & 0 & NA & 17/17 (100) & NR \\
\hline $22 / 93(24)$ & NR & 11/93 (12) & 11/93 (12) & $0 / 93$ & NR & $22 / 93(24)$ & NR \\
\hline $37 / 93(41)$ & NR & $13 / 91(14)$ & $26 / 91(29)$ & $0 / 91$ & NR & NR & NR \\
\hline NR & NR & $\begin{array}{c}7 / 121(11) \\
1 / 60(2)\end{array}$ & NR & NR & NR & NR & NR \\
\hline NR & NR & NR & $<10$ & NR & NR & NR & NR \\
\hline NR & NR & NR & $26 / 46(56)$ & $0 / 46$ & NR & NR & NR \\
\hline NR & NR & $6747(13)$ & NR & $0 / 47$ & NR & NR & NR \\
\hline NR & NR & $8 / 18(44)$ & NR & $0 / 18$ & NR & NA & NR \\
\hline NR & NR & NR & 2/44 (5) & NR & NR & NR & NR \\
\hline NR & NR & 10/139 (7) & $8 / 130(6)$ & NR & NR & NR & NR \\
\hline $8 / 11$ & NR & NR & $\begin{array}{l}8 / 11 \text { (thyroidectomized } \\
\text { patients) }\end{array}$ & $0 / 11$ & $\begin{array}{l}\text { Within } \\
2 \text { weeks }\end{array}$ & $8 / 11$ & NR \\
\hline $2 / 8$ & NR & $1 / 8(13)$ & 0 & $1 / 8(13)$ & Day 7 & $0 / 9$ & NR \\
\hline $30 / 55$ & NR & 6/55 (11) & 6/55 (11) & $18 / 55(33)$ & $\begin{array}{l}248(22-944) \\
\text { days }\end{array}$ & & NR \\
\hline $7 / 10$ & NR & $4 / 10(40)$ & $1 / 10(10)$ & $2 / 10(20)$ & $\begin{array}{l}142(7-370) \\
\text { days }\end{array}$ & & NR \\
\hline
\end{tabular}


Finally, it has been shown that inhibition of angiogenesis might impair peripheral metabolism of thyroid hormones. In rats, the expression of the DIO3 gene increases under hypoxic-ischemic condition via the activation of the hypoxiainducible factor-dependent pathway (210). If this observation is also confirmed in humans, difference in the hypothyroidism rate seen with sunitinib compared with motesanib and vandetanib may be further explained by the different affinity of each drug for the kinases of angiogenic receptors (194). Indeed, motesanib targets PDGFR with far less affinity than the VEGFR2, and vandetanib targets neither VEGFR1 nor PDGFR, while sunitinib showed the highest affinity for VEGFR2 and PDGFR (194).

\section{Bexarotene}

Bexarotene is a selective agonist of the retinoid $X$ receptor, a nuclear hormone receptor with pleiotropic functions on cell growth, apoptosis, and cell differentiation $(211,212)$. Bexarotene is currently approved for treatment of cutaneous T-cell lymphoma (213) and is under investigation for other cancers and autoimmune diseases (214). Bexarotene causes a rapid (4-8 hours) development of central hypothyroidism in $40 \%$ $100 \%$ of patients (215-217). TSH levels return to normal within a few days of drug discontinuation (213). Bexarotene interferes with the pituitary-thyroid axis at different levels. The normal feedback of thyroid hormone on the pituitary is impaired by bexarotene $(216,218)$, which renders the transcription of the TSH $\beta$-subunit gene T3-independent $(216,218)$. Additionally, bexarotene directly inhibits the secretion of TSH (219), explaining the rapid fall in TSH levels after administration of the drug. Bexarotene also affects thyroid hormone metabolism. In thyroidectomized thyroid cancer patients under LT4 who receive bexarotene a marked increase in LT4 requirement is needed. In these patients, bexarotene induces a dramatic decrease in thyroid hormones without compensatory elevation of TSH levels (220), presumably by interfering with peripheral thyroid hormone metabolism via nondeiodinase mechanisms. In experimental studies the drug has induced liver cytochrome P450 systems that also metabolize thyroid hormones (221).

\section{Why Is It Important to Assess Thyroid Function in Cancer Patients?}

Obviously, the correct and timely identification of thyroid dysfunction induced by anticancer drugs is important in obtaining significant diagnostic and therapeutic advantages. However, in cancer patients the recognition of symptoms due to thyroid dysfunction may be difficult. For example, symptoms such as fatigue and/or constipation may be caused not only by underlying malignancy, anticancer treatment(s), or medications used to control other symptoms (i.e., nausea or pain), but also by drug-induced hypothyroidism. Similarly, symptoms of thyrotoxicosis (i.e., palpitations, weight loss, heat intolerance, tremor, proximal muscle weakness, tachycardia, insomnia, irritability, fever) overlap with other complications (e.g., infection/sepsis). Missing the diagnosis of thyroid dysfunction induced by an anticancer drug may lead to unjustified dose reduction or treatment withdrawal of the drug (6). Moreover, untreated hypothyroidism or thyrotoxicosis may affect the metabolism of other medications, including anticancer drugs themselves, potentially reducing their efficacy (222). Undetected thyroid diseases can trigger life-threatening consequences, such as cardiac toxicity presenting as complication of TKI-induced hypothyroidism or eventually myxedematous coma (223-225). The early diagnosis of hypophysitis allows for the detection of central hypothyroidism and for the prevention of other consequences of hypopituitarism, including a life-threatening condition such as adrenal insufficiency (110).

Notably, it has been suggested that pretreatment hypothyroidism or drug-induced thyroid dysfunction may have a prognostic value in cancer patients. Hypothyroidism has been associated with reduced breast cancer incidence, older age at diagnosis, and less aggressive disease (226). However, correlations between hypothyroidism/thyroid autoimmunity and breast cancer remain conflicting (227). Hypothyroidism following RT is a predictor of better survival in head and neck cancer patients (228). Propylthiouracil-induced hypothyroidism was associated with improved survival in patients with high-grade glioma (229). IFN- and IL2-induced thyroid autoimmunity correlated with better prognosis in various studies $(64,65,81,83)$, but not when guarantee-bias survival analyses were performed $(39,49,60)$. In renal cell carcinoma patients receiving sorafenib/sunitinib, better clinical outcomes were seen when hypothyroidism developed $(158,159,168,230)$. Interestingly, higher risk of hypothyroidism during sunitinib therapy has been significantly associated with VEGF-A/VEGFR-2 gene single-nucleotide polymorphisms (152), suggesting that TKIinduced thyroid dysfunction may depend on a specific genetic predisposition. However, in this study better survival did not correlate with TKI-induced hypothyroidism. Retrospective analyses suggest that patients who experience immune-related adverse events, including endocrinopathies, may be more likely to benefit from anti-CTLA4-MAb therapy $(107,231,232)$.

\section{Therapeutic Challenges}

The above issues emphasize that oncologists should maintain adequate surveillance of thyroid function in cancer patients. When drugs that are potentially associated with thyroid side effects are used, thyroid function should be carefully assessed at baseline and monitored throughout the treatment and follow-up period $(6,233)$. Specific guidelines based on high-level evidence are lacking. However, in clinical practice rational approaches may be a guide in appropriately monitoring and treating thyroid dysfunction induced by anticancer drugs $(6,234,235)$.

Before starting IFN, IL2, DD, thalidomide/lenalidomide, or alemtuzumab, TSH and TPO-Ab should be assessed, followed by TSH measurement every $2-3$ months, if TPO-Ab are positive (6). With negative TPO-Ab, a less frequent TSH and TPO-Ab assessment may be indicated. In the case of de novo hypothyroidism, the withdrawal of the causative drug is generally not required, based on the efficacy of LT4 replacement. Thyrotoxicosis requires the treatment of symptoms with $\beta$-blockers. In the rare cases of drug-induced Graves' disease, methimazole or propylthiouracil may be administered if clinically indicated.

As bexarotene directly affects TSH secretion, thyroid function should be monitored with free T4 rather than TSH levels biweekly for the first 2 months and then every 1-2 months $(236,237)$. Bexarotene-induced hypothyroidism can be prevented by starting high LT4 dosage with the drug. To 
obtain the therapeutic goal of free T4 levels in the normal range, an LT4 dosage up to 2-3 times of the usual $1.6 \mu \mathrm{g} / \mathrm{kg} /$ day may be needed due to the drug-induced thyroid hormone degradation (6).

In patients who are candidates to receive ipilimumab, pretreatment thyroid function tests should include both TSH and free T4, as both secondary and primary thyroid dysfunction may occur. Monitoring of pituitary and thyroid function is required on day 1 of every cycle of treatment (238).

Thyroid dysfunction in patients receiving TKI requires specific considerations. In thyroidectomized patients under adequate LT4 replacement who should be treated with imatinib, motesanib, vandetanib, or axitinib, pretreatment TSH evaluation, followed by monthly monitoring of TSH, is recommended. In these patients, a substantial increase up to the doubling of the LT4 dose on initiation of TKI should be considered $(6,194)$. Once TSH levels are stable, monitoring every 2-3 months is advisable. In patients with normal thyroid function, measurement of thyroid function tests before treatment with sunitinib and then measuring TSH on day 1 of every cycle appears appropriate $(146,234,235)$. Elevated TSH levels measured on day 1 of the cycle (at the end of the 2-week rest period) are more likely to indicate clinically relevant thyroid damage requiring further investigation and, if appropriate, initiation of substitutive therapy $(234,235,239)$. Measurement of TSH at day 28 of the cycle (at the end of the 4 -week sunitinib treatment period) may increase the chances of early detection of thyroid dysfunction that may be subclinical/transient, thus not requiring treatment (234). Specific prospective trials are needed to better define the best timing of thyroid function assessment in patients under sunitinib and other TKI treatment $(235,239)$. However, as treatment with these agents may be prolonged (more than 1-2 years) and the risk of thyroid toxicity seemed to be higher in the first 2-4 cycles, measurements of TSH may be empirically advised on day 1 of cycles $1-4$, and then every $2-3$ cycles $(6,146)$.

Patients with overt hypothyroidism (TSH $>10 \mathrm{mIU} / \mathrm{L}$ ) should receive LT4 with the objective of maintaining TSH within the normal range. This may be achieved by an average starting dose of $1.6 \mu \mathrm{g} / \mathrm{kg} /$ day, except in patients with coronary artery disease or arrhythmias, in whom the dosage should be titrated starting with a lower dose (6).

The treatment of subclinical hypothyroidism (TSH 5-10 $\mathrm{mIU} / \mathrm{L}$ with a normal free T4) is questionable in cancer patients. In healthy population, the correction of subclinical hypothyroidism is discouraged due to a lack of evidence concerning its benefit $(240,241)$. In cancer patients, this may be even more problematic. Some preclinical, epidemiological, and clinical evidence suggests that LT4 replacement therapy may be permissive for tumor growth (242), while T3 obtains this effect only at supraphysiological doses (243). Possible effects of thyroid hormones on cancer cells include the amplification of EGFR, phosphorylation of insulin-like growth factor-1 receptor, stimulation of migration, a direct trophic effect on tumor cells, and angiogenesis and cell-specific antiapoptotic activity $(242,244)$. However, effects of thyroid status on cancer cells may be cell type specific (245) and current experimental data need further prospective confirmation.

However, LT4 may be offered to cancer patients presenting with TKI-induced subclinical hypothyroidism and TAb, hypercholesterolemia, thyroid nodules, or symptoms, such as fatigue, that may greatly worsen patients' quality of life.
Notably, in one study (185), at least half of patients who started LT4 for sunitinib-associated hypothyroidism had improvement in fatigue (185). Based on preclinical evidence showing that physiological doses of $\mathrm{T} 4$, but not $\mathrm{T} 3$, contribute to tumor growth, it has been recently reported that selective T3 supplementation in cancer patients may reduce the suggested risk of T4-induced tumor growth (246).

\section{Conclusions}

Thyroid dysfunction is emerging as a variably common endocrine toxicity of several anticancer drugs. However, the pathophysiology of thyroid toxicity induced by these agents remains to be fully clarified. Thyroid side effects induced by anticancer agents are generally manageable and dose reduction or discontinuation of the causative drugs is not required. Routine testing for thyroid abnormalities in patients receiving many of the current anticancer agents is recommendable at baseline, during the treatment, and the period of follow-up. Furthermore, thyroid function tests should be included in routine toxicity assessment of new TKIs and possibly of other classes of targeted drugs under clinical evaluation. The prognostic relevance of thyroid autoimmunity, overt and subclinical hypothyroidism induced by anticancer drugs, the value and the safety of thyroid hormone replacement in individuals with abnormal TSH following anticancer systemic therapy, and the correct timing of replacement therapy need to be defined more accurately. Additional prospective clinical trials are necessary to investigate these important aspects. These trials would offer a unique opportunity to clarify the underlying molecular mechanism of thyroid toxicities induced by an increasing number of anticancer agents and to find potentially predictive factors of thyroid toxicity in cancer patients.

\section{Author Disclosure Statement}

The authors declare that no competing financial interests exist.

\section{References}

1. Wartofsky L, Burman KD 1982 Alterations in thyroid function in patients with systemic illness: the "euthyroid sick syndrome." Endocr Rev 3:164-217.

2. van der Molen AJ, Thomsen HS, Morcos SK 2004 Effect of iodinated contrast media on thyroid function in adults. Eur Radiol 14:902-907.

3. Markou K, Georgopoulos N, Kyriazopoulou V, Vagenakis AG 2001 Iodine-induced hypothyroidism. Thyroid 11:501510.

4. Schneider AB, Sarne DH 2005 Long-term risks for thyroid cancer and other neoplasms after exposure to radiation. Nat Clin Pract Endocrinol Metab 1:82-91.

5. Yeung SC, Chiu AC, Vassilopoulou-Sellin R, Gagel RF 1998 The endocrine effects of nonhormonal antineoplastic therapy. Endocr Rev 19:144-172.

6. Hamnvik OP, Larsen PR, Marqusee E 2011 Thyroid dysfunction from antineoplastic agents. J Natl Cancer Inst 103:1572-1587.

7. Bruning PF, Bonfrèr JG, Engelsman E, Hamersma-vd Linden E, de Jong-Bakker M, Nooyen W 1984 Pros and cons of aminoglutethimide for advanced postmenopausal breast cancer. Breast Cancer Res Treat 4:289-295. 
8. Figg WD, Thibault A, Sartor AO, Mays D, Headlee D, Calis KA, Cooper MR 1994 Hypothyroidism associated with aminoglutethimide in patients with prostate cancer. Arch Intern Med 154:1023-1025.

9. Stava CJ, Jimenez C, Vassilopoulou-Sellin R 2007 Endocrine sequelae of cancer and cancer treatments. J Cancer Surviv 1:261-274.

10. Massart C, Le Tellier C, Lucas C, Gibassier J, Leclech G, Nicol M 1992 Effects of cisplatin on human thyrocytes in monolayer or follicle culture. J Mol Endocrinol 8:243-248.

11. Beex L, Ross A, Smals A, Kloppenborg P 1977 5-fluorouracil-induced increase of total serum thyroxine and triiodothyronine. Cancer Treat Rep 61:1291-1295.

12. Surks MI, Sievert R 1995 Drugs and thyroid function. N Engl J Med 333:1688-1694.

13. Dong BJ 2000 How medications affect thyroid function. West J Med 172:102-106.

14. Mamby CC, Love RR, Lee KE 1995 Thyroid function test changes with adjuvant tamoxifen therapy in postmenopausal women with breast cancer. J Clin Oncol 13:854-857.

15. Garnick MB, Larsen PR 1979 Acute deficiency of thyroxinebinding globulin during L-asparaginase therapy. N Engl J Med 301:252-253.

16. Ferster A, Glinoer D, Van Vliet G, Otten J 1992 Thyroid function during L-asparaginase therapy in children with acute lymphoblastic leukemia: difference between induction and late intensification. Am J Pediatr Hematol Oncol 14:192-196.

17. Heidemann PH, Stubbe P, Beck W 1981 Transient secondary hypothyroidism and thyroxine binding globulin deficiency in leukemic children during polychemotherapy: an effect of L-asparaginase. Eur J Pediatr 136:291-295.

18. Zatelli MC, Gentilin E, Daffara F, Tagliati F, Reimondo G, Carandina G, Ambrosio MR, Terzolo M, Degli Uberti EC 2010 Therapeutic concentrations of mitotane [o,p'-DDD] inhibit thyrotroph cell viability and TSH expression and secretion in a mouse cell line model. Endocrinology 151:2453-2461.

19. Daffara F, De Francia S, Reimondo G, Zaggia B, Aroasio E, Porpiglia F, Volante M, Termine A, Di Carlo F, Dogliotti L, Angeli A, Berruti A, Terzolo M 2008 Prospective evaluation of mitotane toxicity in adrenocortical cancer patients treated adjuvantly. Endocr Relat Cancer 15:1043-1053.

20. Stuart NS, Woodroffe CM, Grundy R, Cullen MH 1990 Long-term toxicity of chemotherapy for testicular cancerthe cost of cure. Br J Cancer 61:479-484.

21. Ssutcliffe SB, Chapman R, Wrigley PF 1981 Cyclical combination chemotherapy and thyroid function in patients with advanced Hodgkin's disease. Med Pediatr Oncol 9:439-448.

22. Hancock SL, Cox RS, McDougall IR 1991 Thyroid diseases after treatment of Hodgkin's disease. $N$ Engl J Med 325:599-605.

23. Ogilvy-Stuart AL, Shalet SM, Gattamaneni HR 1991 Thyroid function after treatment of brain tumors in children. J Pediatr 119:733-737.

24. Paulino RC 2002 Hypothyroidism in children with medulloblastoma: a comparison of 3600 and 2340 cGy craniospinal radiotherapy. Int J Radiat Oncol Biol Phys 53:543-547.

25. Kirkwood JM 1991 Studies of interferons in the therapy of melanoma. Semin Oncol 18 (5 Suppl 7):83-90.

26. Jonasch E, Haluska FG 2001 Interferon in oncological practice: review of interferon biology, clinical applications, and toxicities. Oncologist 6:34-55.
27. Friedman RM 2008 Clinical uses of interferons. Br J Clin Pharmacol 65:158-162.

28. Mandac JC, Chaudhry S, Sherman KE, Tomer Y 2006 The clinical and physiological spectrum of interferon-alpha induced thyroiditis: toward a new classification. Hepatology 43:661-672.

29. Tomer Y, Blackard JT, Akeno N 2007 Interferon alpha treatment and thyroid dysfunction. Endocrinol Metab Clin North Am 36:1051-1066.

30. www.merck.com/product/usa/pi_circulars/i/intron_a/ intron_a_pi.pdf Accessed April 13, 2013.

31. Koh LK, Greenspan FS, Yeo PP 1997 Interferon-alpha induced thyroid dysfunction: three clinical presentations and a review of the literature. Thyroid 7:891-896.

32. Vial T, Choquet-Kastylevsky G, Liautard C 2000 Endocrine and neurological adverse effects of the therapeutic interferons. Toxicology 142:161-172.

33. Chan WB, Chow CC, Cockram CS 2003 Interferon alpha treatment and endocrine disease. J R Soc Med 96: 481-485.

34. Carella C, Mazziotti G, Amato G, Braverman LE, Roti E 2004 Interferon-a-related thyroid disease: pathophysiological, epidemiological, and clinical aspects. J Clin Endocrinol Metab 89:3656-3661.

35. Monzani F, Caraccio N, Dardano A, Ferrannini E 2004 Thyroid autoimmunity and dysfunction associated with interferon therapy. Clin Exp Med 3:199-210.

36. Gogas H, Ioannovich J, Dafni U, Stavropoulou-Giokas C, Frangia K, Tsoutsos D, Panagiotou P, Polyzos A, Papadopoulos O, Stratigos A, Markopoulos C, Bafaloukos D, Pectasides D, Fountzilas G, Kirkwood JM 2006 Prognostic significance of autoimmunity during treatment of melanoma with interferon. N Engl J Med 354:709-718.

37. Satzger I, Meier A, Schenck F, Kapp A, Hauschild A, Gutzmer R 2007 Autoimmunity as a prognostic factor in melanoma patients treated with adjuvant low-dose interferon alpha. Int J Cancer 121:2562-2566.

38. Hauschild A, Gogas H, Tarhini A, Middleton MR, Testori A, Dréno B, Kirkwood JM 2008 Practical guidelines for the management of interferon-alpha-2b side effects in patients receiving adjuvant treatment for melanoma: expert opinion. Cancer 112:982-994.

39. Bouwhuis MG, Ten Hagen TL, Suciu S, Eggermont AM 2011 Autoimmunity and treatment outcome in melanoma. Curr Opin Oncol 23:170-176.

40. Fentiman IS, Balkwill FR, Thomas BS, Russell MJ, Todd I, Bottazzo GF 1988 An autoimmune aetiology for hypothyroidism following interferon therapy for breast cancer. Eur J Cancer Clin Oncol 24:1299-1303.

41. Ronnblom LE, Alm GV, Oberg KE 1991 Autoimmunity after alpha interferon therapy for malignant carcinoid tumors. Ann Intern Med 115:178-183.

42. Silvestri F, Virgolini L, Mazzolini A, Bertolissi F, Russo D, Fanin R, Baccarani M 1994 Development of autoimmune thyroid diseases during long-term treatment of hematological malignancies with alpha-interferons. Haematologica 79:367-370.

43. Vallisa D, Cavanna L, Berte R, Merli F, Ghisoni F, Buscarini L 1995 Autoimmune thyroid dysfunctions in hematologic malignancies treated with alpha-interferon. Acta Haematol 93:31-35.

44. Fattovich G, Giustina G, Favarato S, Ruol A 1996 A survey of adverse events in 11,241 patients with chronic viral hepatitis treated with alfa interferon. J Hepatol 24:38-47. 
45. Tran HA, Jones TL, Ianna EA, Reeves GE 2011 The natural history of interferon-alpha induced thyroiditis in chronic hepatitis C patients: a long term study. Thyroid Res 4:2.

46. Marazuela M, Garcia-Buey L, Gonzalez-Fernandez B, García-Monzón C, Arranz A, Borque MJ, Moreno-Otero R 1996 Thyroid autoimmune disorders in patients with chronic hepatitis $\mathrm{C}$ before and during interferon-alpha therapy. Clin Endocrinol (Oxf) 44:635-642.

47. Deutsch M, Dourakis S, Manesis EK, Gioustozi A, Hess G, Horsch A, Hadziyannis S 1997 Thyroid abnormalities in chronic viral hepatitis and their relationship to interferon alfa therapy. Hepatology 26:206-210.

48. Roti E, Minelli R, Giuberti T, Marchelli S, Schianchi C, Gardini E, Salvi M, Fiaccadori F, Ugolotti G, Neri TM, Braverman LE 1996 Multiple changes in thyroid function in patients with chronic active HCV hepatitis treated with recombinant interferon-alpha. Am J Med 101:482-487.

49. Bouwhuis MG, Suciu S, Collette S, Aamdal S, Kruit WH, Bastholt L, Stierner U, Salès F, Patel P, Punt CJ, Hernberg M, Spatz A, ten Hagen TL, Hansson J, Eggermont AM 2009 Autoimmune antibodies and recurrence-free interval in melanoma patients treated with adjuvant interferon. J Natl Cancer Inst 101:869-877.

50. Silvestri F, Virgolini L, Mazzolini A, Bertolissi F, Russo D, Fanin R, Baccarani M 1994 Development of autoimmune thyroid diseases during long-term treatment of hematological malignancies with alpha-interferons. Haematologica 79:367-370.

51. Pfeffer LM, Dinarello CA, Herberman RB, Williams BR, Borden EC, Bordens R, Walter MR, Nagabhushan TL, Trotta PP, Pestka S 1998 Biological properties of recombinant alpha-interferons: 40th anniversary of the discovery of interferons. Cancer Res 58:2489-2499.

52. Corssmit EP, de Metz J, Sauerwein HP, Romijn JA 2000 Biologic responses to IFN-alpha administration in humans. J Interferon Cytokine Res 20:1039-1047.

53. Krause I, Valesini G, Scrivo R, Shoenfeld Y 2003 Autoimmune aspects of cytokine and anticytokine therapies. Am J Med 115:390-397.

54. Lindahl P, Leary P, Gresser I 1973 Enhancement by interferon of the expression of surface antigens on murine leukemia L 1210 cells. Proc Natl Acad Sci USA 70:2785-2788.

55. Yue SJ, Enomoto T, Matsumoto Y, Kawai K, Volpe R 1998 Thyrocyte class I and class II upregulation is a secondary phenomenon and does not contribute to the pathogenesis of autoimmune thyroid disease. Thyroid 8:755-763.

56. Farrar JD, Murphy KM 2000 Type I interferons and T helper development. Immunol Today 21:484-489.

57. Tilg H $1997 \mathrm{New}$ insights into the mechanisms of interferon alfa: an immunoregulatory and anti-inflammatory cytokine. Gastroenterology 112:1017-1021.

58. Caraccio N, Giannini R, Cuccato S, Faviana P, Berti P, Galleri D, Dardano A, Basolo F, Ferrannini E, Monzani F 2005 Type I interferons modulate the expression of thyroid peroxidase, sodium/iodide symporter, and thyroglobulin genes in primary human thyrocyte cultures. J Clin Endocrinol Metab 90:1156-1162.

59. Glue P, Fang JW, Rouzier-Panis R, Raffanel C, Sabo R, Gupta SK, Salfi M, Jacobs S 2000 Pegylated interferonalpha2b: pharmacokinetics, pharmacodynamics, safety, and preliminary efficacy data. Hepatitis C Intervention Therapy Group. Clin Pharmacol Ther 68:556-567.

60. Eggermont AM, Bouwhuis MG, Kruit WH, Testori A, ten Hagen T, Yver A, Xu C 2010 Serum concentrations of pegylated interferon alpha- $2 b$ in patients with resected stage III melanoma receiving adjuvant pegylated interferon alpha-2b in a randomized phase III trial (EORTC 18991). Cancer Chemother Pharmacol 65:671-677.

61. Bukowski RM, Tendler C, Cutler D, Rose E, Laughlin MM, Statkevich P 2002 Treating cancer with PEG Intron: pharmacokinetic profile and dosing guidelines for an improved interferonalpha-2b formulation. Cancer 95:389-396.

62. Eggermont AM, Suciu S, Santinami M, Testori A, Kruit WH, Marsden J, Punt CJ, Salès F, Gore M, Mackie R, Kusic Z, Dummer R, Hauschild A, Musat E, Spatz A, Keilholz U; EORTC Melanoma Group 2008 Adjuvant therapy with pegylated interferon alfa- $2 \mathrm{~b}$ versus observation alone in resected stage III melanoma: final results of EORTC 18991, a randomised phase III trial. Lancet 372:117-126.

63. Bouwhuis MG, Suciu S, Testori A, Kruit WH, Salès F, Patel P, Punt CJ, Santinami M, Spatz A, Ten Hagen TL, Eggermont AM 2010 Phase III trial comparing adjuvant treatment with pegylated interferon Alfa- $2 b$ versus observation: prognostic significance of autoantibodies-EORTC 18991. J Clin Oncol 28:2460-2466.

64. Weijl NI, Van der Harst D, Brand A, Kooy Y, Van Luxemburg S, Schroder J, Lentjes E, Van Rood JJ, Cleton FJ, Osanto S 1993 Hypothyroidism during immunotherapy with interleukin-2 is associated with antithyroid antibodies and response to treatment. J Clin Oncol 11:1376-1383.

65. Atkins MB, Mier JW, Parkinson DR, Gould JA, Berkman EM, Kaplan MM 1988 Hypothyroidism after treatment with interleukin-2 and lymphokine-activated killer cells. N Engl J Med 318:1557-1563.

66. Fraenkel PG, Rutkove SB, Matheson JK, Fowkes M, Cannon ME, Patti ME, Atkins MB, Gollob JA 2002 Induction of myasthenia gravis, myositis, and insulin-dependent diabetes mellitus by high-dose interleukin-2 in a patient with renal cell cancer. J Immunother 25:373-378.

67. Krouse RS, Royal RE, Heywood G, Weintraub BD, White DE, Steinberg SM, Rosenberg SA, Schwartzentruber DJ 1995 Thyroid dysfunction in 281 patients with metastatic melanoma or renal carcinoma treated with interleukin-2 alone. J Immunother Emphasis Tumor Immunol 18:272-278.

68. Vialettes B, Guillerand MA, Viens P, Stoppa AM, Baume D, Sauvan R, Pasquier J, San Marco M, Olive D, Maraninchi D 1993 Incidence rate and risk factors for thyroid dysfunction during recombinant interleukin-2 therapy in advanced malignancies. Acta Endocrinol (Copenh) 129:31-38.

69. Schwartzentruber DJ, White DE, Zweig MH, Weintraub BD, Rosenberg SA 1991 Thyroid dysfunction associated with immunotherapy for patients with cancer. Cancer 68: 2384-2390.

70. Jacobs EL, Clare-Salzler MJ, Chopra IJ, Figlin RA 1991 Thyroid function abnormalities associated with the chronic outpatient administration of recombinant interleukin-2 and recombinant interferon-alpha. J Immunother 10:448-455.

71. Chianese-Bullock KA, Woodson EM, Tao H, Boerner SA, Smolkin M, Grosh WW, Neese PY, Merrill P, Petroni GR, Slingluff CL Jr 2005 Autoimmune toxicities associated with the administration of antitumor vaccines and low-dose interleukin-2. J Immunother 28:412-419.

72. Vassilopoulou-Sellin R, Sella A, Dexeus FH, Theriault RL, Pololoff DA 1992 Acute thyroid dysfunction [thyroiditis] after therapy with interleukin-2. Horm Metab Res 24:434-438.

73. Pichert G, Jost LM, Zobeli L, Odermatt B, Pedia G, Stahel RA 1990 Thyroiditis after treatment with interleukin-2 and interferon alpha-2a. Br J Cancer 62:100-104. 
74. Scalzo S, Gengaro A, Boccoli G, Masciulli R, Giannella G, Salvo G, Marolla P, Carlini P, Massimini G, Holdener EE 1990 Primary hypothyroidism associated with interleukin-2 and interferon alpha-2 therapy of melanoma and renal carcinoma. Eur J Cancer 26:1152-1156.

75. Numerof RP, Aronson FR, Mier JW 1988 IL-2 stimulates the production of IL-1 alpha and IL-1 beta by human peripheral blood mononuclear cells. J Immunol 141:4250-4257.

76. Mier JW, Vachino G, van der Meer JW, Numerof RP, Adams S, Cannon JG, Bernheim HA, Atkins MB, Parkinson DR, Dinarello CA 1988 Induction of circulating tumor necrosis factor (TNF alpha) as the mechanism for the febrile response to interleukin-2 (IL-2) in cancer patients. J Clin Immunol 8:426-436.

77. Lotze MT, Matory YL, Ettinghausen SE, Rayner AA, Sharrow SO, Seipp CA, Custer MC, Rosenberg SA 1985 In vivo administration of purified human interleukin 2 II. Half life, immunologic effects, and expansion of peripheral lymphoid cells in vivo with recombinant IL 2. J Immunol 135:2865-2875.

78. Gemlo BT, Palladino MA Jr., Jaffe HS, Espevik TP, Rayner AA 1988 Circulating cytokines in patients with metastatic cancer treated with recombinant interleukin 2 and lymphokineactivated killer cells. Cancer Res 48:5864-5867.

79. Pang XP, Hershman JM, Chung M, Pekary AE 1989 Characterization of tumor necrosis factor-alpha receptors in human and rat thyroid cells and regulation of the receptors by thyrotropin. Endocrinology 125:1783-1788.

80. Kragel AH, Travis WD, Feinberg L, Pittaluga S, Striker LM, Roberts WC, Lotze MT, Yang JJ, Rosenberg SA 1990 Pathologic findings associated with interleukin-2-based immunotherapy for cancer: a postmortem study of 19 patients. Hum Pathol 21:493-502.

81. Franzke A, Peest D, Probst-Kepper M, Buer J, Kirchner GI, Brabant G, Kirchner H, Ganser A, Atzpodien J 1999 Autoimmunity resulting from cytokine treatment predicts long-term survival in patients with metastatic renal cell cancer. J Clin Oncol 17:529-533.

82. Krouse RS, Royal RE, Heywood G, Weintraub BD, White DE, Steinberg SM, Rosenberg SA, Schwartzentruber DJ 1995 Thyroid dysfunction in 281 patients with metastatic melanoma or renal carcinoma treated with interleukin-2 alone. J Immunother Emphasis Tumor Immunol 18:272278.

83. Phan GQ, Attia P, Steinberg SM, White DE, Rosenberg SA 2001 Factors associated with response to high-dose interleukin-2 in patients with metastatic melanoma. J Clin Oncol 19:3477-3482.

84. Kruit WH, Bolhuis RL, Goey SH, Jansen RL, Eggermont AM, Batchelor D, Schmitz PI, Stoter G 1993 Interleukin-2induced thyroid dysfunction is correlated with treatment duration but not with tumor response. J Clin Oncol 11: 921-924.

85. Barbesino G 2010 Drugs affecting thyroid function. Thyroid 20:763-770.

86. Ho VT, Zahrieh D, Hochberg E, Micale E, Levin J, Reynolds C, Steckel S, Cutler C, Fisher DC, Lee SJ, Alyea EP, Ritz J, Soiffer RJ, Antin JH 2004 Safety and efficacy of denileukin diftitox in patients with steroid-refractory acute graftversus-host disease after allogeneic hematopoietic stem cell transplantation. Blood 104:1224-1226.

87. Olsen E, Duvic M, Frankel A, Kim Y, Martin A, Vonderheid E, Jegasothy B, Wood G, Gordon M, Heald P, Oseroff A, Pinter-Brown L, Bowen G, Kuzel T, Fivenson D, Foss F,
Glode M, Molina A, Knobler E, Stewart S, Cooper K, Stevens S, Craig F, Reuben J, Bacha P, Nichols J 2001 Pivotal phase III trial of two dose levels of denileukin diftitox for the treatment of cutaneous T-cell lymphoma. J Clin Oncol 19:376-388.

88. Ghori F, Polder KD, Pinter-Brown LC, Hoff AO, Gagel RF, Sherman SI, Duvic M 2006 Thyrotoxicosis after denileukin diftitox therapy in patients with mycosis fungoides. J Clin Endocrinol Metab 91:2205-2208.

89. Rajkumar SV, Blood E, Vesole D, Fonseca R, Greipp PR; Eastern Cooperative Oncology Group 2006 Phase III clinical trial of thalidomide plus dexamethasone compared with dexamethasone alone in newly diagnosed multiple myeloma: a clinical trial coordinated by the Eastern Cooperative Oncology Group. J Clin Oncol 24:431-436.

90. Shah SR, Tran TM 2007 Lenalidomide in myelodysplastic syndrome and multiple myeloma. Drugs 67:1869-1881.

91. Anderson KC 2005 Lenalidomide and thalidomide: mechanisms of action-similarities and differences. Semin Hematol 42(suppl 4):S3-S8.

92. Badros AZ, Siegel E, Bodenner D, Zangari M, Zeldis J, Barlogie B, Tricot G 2002 Hypothyroidism in patients with multiple myeloma following treatment with thalidomide. Am J Med 112:412-413.

93. Figaro MK, Clayton W Jr., Usoh C, Brown K, Kassim A, Lakhani VT, Jagasia S 2011 Thyroid abnormalities in patients treated with lenalidomide for hematological malignancies: results of a retrospective case review. Am J Hematol 86:467-470.

94. List A, Dewald G, Bennett J, Giagounidis A, Raza A, Feldman E, Powell B, Greenberg P, Thomas D, Stone R, Reeder C, Wride K, Patin J, Schmidt M, Zeldis J, Knight R 2006 Lenalidomide in the myelodysplastic syndrome with chromosome 5q deletion. N Engl J Med 355:1456-1465.

95. Dispenzieri A, Lacy MQ, Zeldenrust SR, Hayman SR, Kumar SK, Geyer SM, Lust JA, Allred JB, Witzig TE, Rajkumar SV, Greipp PR, Russell SJ, Kabat B, Gertz MA 2007 The activity of lenalidomide with or without dexamethasone in patients with primary systemic amyloidosis. Blood 109:465-470.

96. Palumbo A, Hajek R, Delforge M, Kropff M, Petrucci MT, Catalano J, Gisslinger H, Wiktor-Jędrzejczak W, Zodelava M, Weisel K, Cascavilla N, Iosava G, Cavo M, Kloczko J, Bladé J, Beksac M, Spicka I, Plesner T, Radke J, Langer C, Ben Yehuda D, Corso A, Herbein L, Yu Z, Mei J, Jacques C, Dimopoulos MA; MM-015 Investigators 2012 Continuous lenalidomide treatment for newly diagnosed multiple myeloma. N Engl J Med 366:1759-1769.

97. Somers GF 1960 Pharmacological properties of thalidomide [alpha-phthalimido glutarimide], a new sedative hypnotic drug. Br J Pharmacol Chemother 15:111-116.

98. Murdoch JM, Campbell GD 1958 Antithyroid activity of Nphthalyl glutamic acid imide [K17]. Br Med J 1:84-85.

99. Menon S, Habermann T, Witzig T 2007 Lenalidomideassociated hypothyroidism. Leuk Lymphoma 48:2465-2467.

100. Stein EM, Rivera C 2007 Transient thyroiditis after treatment with lenalidomide in a patient with metastatic renal cell carcinoma. Thyroid 17:681-683.

101. Postow M, Callahan MK, Wolchok JD 2011 Beyond cancer vaccines: a reason for future optimism with immunomodulatory therapy. Cancer J 17:372-378.

102. Leach DR, Krummel MF, Allison JP 1996 Enhancement of antitumor immunity by CTLA-4 blockade. Science 271: $1734-1736$. 
103. Sharma P, Wagner K, Wolchok JD, Allison JP 2011 Novel cancer immunotherapy agents with survival benefit: recent successes and next steps. Nat Rev Cancer 11:805-812.

104. Tarhini A, Lo E, Minor DR 2010 Releasing the brake on the immune system: ipilimumab in melanoma and other tumors. Cancer Biother Radiopharm 25:601-613.

105. Hodi FS, O'Day SJ, McDermott DF, Weber RW, Sosman JA, Haanen JB, Gonzalez R, Robert C, Schadendorf D, Hassel JC, Akerley W, van den Eertwegh AJ, Lutzky J, Lorigan $\mathrm{P}$, Vaubel JM, Linette GP, Hogg D, Ottensmeier CH, Lebbe C, Peschel C, Quirt I, Clark JI, Wolchok JD, Weber JS, Tian J, Yellin MJ, Nichol GM, Hoos A, Urba WJ 2010 Improved survival with ipilimumab in patients with metastatic melanoma. N Engl J Med 363:711-723.

106. Di Giacomo AM, Biagioli M, Maio M 2010 The emerging toxicity profiles of anti-CTLA-4 antibodies across clinical indications. Semin Oncol 37:499-507.

107. Attia P, Phan GQ, Maker AV, Robinson MR, Quezado MM, Yang JC, Sherry RM, Topalian SL, Kammula US, Royal RE, Restifo NP, Haworth LR, Levy C, Mavroukakis SA, Nichol G, Yellin MJ, Rosenberg SA 2005 Autoimmunity correlates with tumor regression in patients with metastatic melanoma treated with anti-cytotoxic T-lymphocyte antigen-4. J Clin Oncol 23:6043-6053.

108. Yang JC, Hughes M, Kammula U, Royal R, Sherry RM, Topalian SL, Suri KB, Levy C, Allen T, Mavroukakis S, Lowy I, White DE, Rosenberg SA 2007 Ipilimumab (antiCTLA4 antibody) causes regression of metastatic renal cell cancer associated with enteritis and hypophysitis. J Immunother 30:825-830.

109. Kaehler KC, Piel S, Livingstone E, Schilling B, Hauschild A, Schadendorf D 2010 Update on immunologic therapy with anti-CTLA-4 antibodies in melanoma: identification of clinical and biological response patterns, immune-related adverse events, and their management. Semin Oncol 37: 485-498.

110. Torino F, Barnabei A, De Vecchis L, Salvatori R, Corsello SM 2012 Hypophysitis induced by monoclonal antibodies to cytotoxic T lymphocyte antigen 4: challenges from a new cause of a rare disease. Oncologist 17:525-535.

111. Juszczak A, Gupta A, Karavitaki N, Middleton MR,Grossman AB 2012 Ipilimumab: a novel immunomodulating therapy causing autoimmune hypophysitis: a case report and review. Eur J Endocrinol 167:1-5.

112. Corsello SM, Barnabei A, Marchetti P, De Vecchis L, Salvatori R, Torino F 2013 Endocrine side effects induced by immune checkpoint inhibitors. J Clin Endocrinol Metab 98:1361-1375.

113. Ribas A, Hauschild A, Kefford R, Punt CJ, Haanen JB, Marmol M, Garbe C, Gomez-Navarro J, Pavlov D, Marshall M 2008 Phase III, open-label, randomized, comparative study of tremelimumab (CP-675,206) and chemotherapy (temozolomide [TMZ] or dacarbazine [DTIC]) in patients with advanced melanoma. J Clin Oncol 26:9011 (abstract).

114. Kirkwood JM, Lorigan P, Hersey P, Hauschild A, Robert C, McDermott D, Marshall MA, Gomez-Navarro J, Liang JQ, Bulanhagui CA 2010 Phase II trial of tremelimumab (CP$675,206)$ in patients with advanced refractory or relapsed melanoma. Clin Cancer Res 16:1042-1048.

115. Di Giacomo AM, Danielli R, Calabrò L, Bertocci E, Nannicini C, Giannarelli D, Balestrazzi A, Vigni F, Riversi V, Miracco C, Biagioli M, Altomonte M, Maio M 2011 Ipilimumab experience in heavily pretreated patients with melanoma in an expanded access program at the Uni- versity Hospital of Siena (Italy). Cancer Immunol Immunother 60:467-477.

116. Hodi FS, Friedlander PA, Atkins MB, McDermott DF, Lawrence DP, Ibrahim N, Wu X, Zhou J, Giobbie-Hurder A, Murphy G, Hollman T, Velazquez E, Russell S, Dipiro P, Yap JT, Van Den Abbeele AD 2011 A phase I trial of ipilimumab plus bevacizumab in patients with unresectable stage III or stage IV melanoma. J Clin Oncol 29:8511 (abstract).

117. van den Eertwegh AJ, Versluis J, van den Berg HP 2012 Combined immunotherapy with granulocyte-macrophage colony-stimulating factor-transduced allogeneic prostate cancer cells and ipilimumab in patients with metastatic castration-resistant prostate cancer: a phase 1 dose-escalation trial. Lancet Oncol 13:509-517.

118. Maker AV, Yang JC, Sherry RM, Topalian SL, Kammula US, Royal RE, Hughes M, Yellin MJ, Haworth LR, Levy C, Allen T, Mavroukakis SA, Attia P, Rosenberg SA 2006 Intrapatient dose escalation of anti-CTLA-4 antibody in patients with metastatic melanoma. J Immunother 29:455-463.

119. Min L, Vaidya A, Becker C 2011 Thyroid autoimmunity and ophthalmopathy related to melanoma biological therapy. Eur J Endocrinol 164:303-307.

120. Borodic G, Hinkle DM, Cia Y 2011 Drug-induced Graves disease from ctla-4 receptor suppression. Ophthal Plast Reconstr Surg 27:e87-e88.

121. Ascierto PA, Simeone E, Sznol M, Fu YX, Melero I 2010 Clinical experiences with anti-CD137 and anti-PD1 therapeutic antibodies. Semin Oncol 37:508-516.

122. Topalian SL, Hodi FS, Brahmer JR, Gettinger SN, Smith DC, McDermott DF, Powderly JD, Carvajal RD, Sosman JA, Atkins MB, Leming PD, Spigel DR, Antonia SJ, Horn L, Drake CG, Pardoll DM, Chen L, Sharfman WH, Anders RA, Taube JM, McMiller TL, Xu H, Korman AJ, Jure-Kunkel M, Agrawal S, McDonald D, Kollia GD, Gupta A, Wigginton JM, Sznol M 2012 Safety, activity, and immune correlates of anti-PD-1 antibody in cancer. N Engl J Med 366:2443-2454.

123. Brahmer JR, Tykodi SS, Chow LQ, Hwu WJ, Topalian SL, Hwu P, Drake CG, Camacho LH, Kauh J, Odunsi K, Pitot HC, Hamid O, Bhatia S, Martins R, Eaton K, Chen S, Salay TM, Alaparthy S, Grosso JF, Korman AJ, Parker SM, Agrawal S, Goldberg SM, Pardoll DM, Gupta A, Wigginton JM 2012 Safety and activity of anti-PD-L1 antibody in patients with advanced cancer. N Engl J Med 366:2455-2465.

124. Lockwood CM, Hale G, Waldman H, Jayne DR 2003 Remission induction in Behcet's disease following lymphocyte depletion by the anti-CD52 antibody CAMPATH $1-\mathrm{H}$. Rheumatology (Oxford) 42:1539-1544.

125. Campath Product Label. www.accessdata.fda.gov/ drugsatfda_docs/label/2007/103948s5070lbl.pdf Accessed April 13, 2013.

126. Coles AJ, Compston DA, Selmaj KW, Lake SL, Moran S, Margolin DH, Norris K, Tandon PK 2008 Alemtuzumab vs. interferon beta-1a in early multiple sclerosis. N Engl J Med 359:1786-1801.

127. Shaw BE, Apperley JF, Russell NH, Craddock C, Liakopoulou E, Potter MN, Wynn R, Gibson B, Pearce RM, Kirkland K, Lee J, Madrigal JA, Cook G, Byrne JL 2011 Unrelated donor peripheral blood stem cell transplants incorporating pre-transplant in-vivo Alemtuzumab are not associated with any increased risk of significant acute or chronic graft-versus-host disease. Br J Haematol 153: 244-252. 
128. Kanda J, Lopez RD, Rizzieri DA 2011 Alemtuzumab for the prevention and treatment of graft-versus-host disease. Int J Hematol 93:586-593.

129. Kirk AD, Hale DA, Swanson SJ, Mannon RB 2006 Autoimmune thyroid disease after renal transplantation using depletional induction with alemtuzumab. Am J Transplant 6:1084-1085.

130. Walsh M, Chaudhry A, Jayne D 2008 Long-term follow-up of relapsing/refractory anti-neutrophil cytoplasm antibody associated vasculitis treated with the lymphocyte depleting antibody alemtuzumab (CAMPATH-1H). Ann Rheum Dis 67:1322-1327.

131. Lorenzi AR, Clarke AM, Wooldridge T, Waldmann H, Hale G, Symmons D, Hazleman BL, Isaacs JD 2008 Morbidity and mortality in rheumatoid arthritis patients with prolonged therapy-induced lymphopenia: twelve-year outcomes. Arthritis Rheum 58:370-375.

132. Demko S, Summers J, Keegan P, Pazdur R 2008 FDA drug approval summary: alemtuzumab as single-agent treatment for B-cell chronic lymphocytic leukemia. Oncologist 13:167-174.

133. Coles AJ, Wing M, Smith S, Coraddu F, Greer S, Taylor C, Weetman A, Hale G, Chatterjee VK, Waldmann $H$, Compston A 1999 Pulsed monoclonal antibody treatment and autoimmune thyroid disease in multiple sclerosis. Lancet 354:1691-1695.

134. Coles AJ, Cox A, Le Page E, Jones J, Trip SA, Deans J, Seaman S, Miller DH, Hale G, Waldmann H, Compston DA 2006 The window of therapeutic opportunity in multiple sclerosis: evidence from monoclonal antibody therapy. J Neurol 253:98-108.

135. Milenic DE, Brady ED, Brechbiel MW 2004 Antibodytargeted radiation cancer therapy. Nature Rev Drug Discovery 3:488-498.

136. Gopal AK, Rajendran JG, Gooley TA, Pagel JM, Fisher DR, Petersdorf SH, Maloney DG, Eary JF, Appelbaum FR, Press OW 2007 High-dose [131I]tositumomab (anti-CD20) radioimmunotherapy and autologous hematopoietic stemcell transplantation for adults $>$ or $=60$ years old with relapsed or refractory B-cell lymphoma. J Clin Oncol 25:13961402.

137. Press OW, Unger JM, Braziel RM, Maloney DG, Miller TP, Leblanc M, Fisher RI 2006 Phase II trial of CHOP chemotherapy followed by tositumomab/iodine I-131 tositumomab for previously untreated follicular non-Hodgkin's lymphoma: five-year follow-up of Southwest Oncology Group Protocol S9911. J Clin Oncol 24:4143-4149.

138. Kaminski MS, Tuck M, Estes J, Kolstad A, Ross CW, Zasadny K, Regan D, Kison P, Fisher S, Kroll S, Wahl RL 2005 131I-tositumomab therapy as initial treatment for follicular lymphoma. N Engl J Med 352:441-449.

139. Quach A, Ji L, Mishra V, Sznewajs A, Veatch J, Huberty J, Franc B, Sposto R, Groshen S, Wei D, Fitzgerald P, Maris JM, Yanik G, Hawkins RA, Villablanca JG, Matthay KK 2011 Thyroid and hepatic function after high-dose 131 Imetaiodobenzylguanidine (131 I-MIBG) therapy for neuroblastoma. Pediatr Blood Cancer 56:191-201.

140. de Kraker J, Hoefnagel KA, Verschuur AC, van Eck B, van Santen HM, Caron HN 2008 Iodine-131-metaiodobenzylguanidine as initial induction therapy in stage 4 neuroblastoma patients over 1 year of age. Eur J Cancer 44:551-556.

141. van Santen HM, de Kraker J, van Eck BL, de Vijlder JJ, Vulsma T 2002 High incidence of thyroid dysfunction de- spite prophylaxis with potassium iodide during (131)Imeta-iodobenzylguanidine treatment in children with neuroblastoma. Cancer 94:2081-2089.

142. Fabbro D, Cowan-Jacob SW, Möbitz H, Martiny-Baron G 2012 Targeting cancer with small-molecular-weight kinase inhibitors. Methods Mol Biol 795:1-34.

143. Lodish MB 2013 Kinase inhibitors: adverse effects related to the endocrine system. J Clin Endocrinol Metab 98:13331342.

144. Grossmann M, Premaratne E, Desai J, Davis ID 2008 Thyrotoxicosis during sunitinib treatment for renal cell carcinoma. Clin Endocrinol (Oxf) 69:669-672.

145. Brown RL 2011 Tyrosine kinase inhibitor-induced hypothyroidism: incidence, etiology, and management. Target Oncol 6:217-226.

146. Wolter P, Stefan C, Decallonne B, Dumez H, Bex M, Carmeliet P, Schöffski P 2008 The clinical implications of sunitinib-induced hypothyroidism: a prospective evaluation. Br J Cancer 99:448-454.

147. Desai J, Yassa L, Marqusee E, George S, Frates MC, Chen $\mathrm{MH}$, Morgan JA, Dychter SS, Larsen PR, Demetri GD, Alexander EK 2006 Hypothyroidism after sunitinib treatment for patients with gastrointestinal stromal tumors. Ann Intern Med 145:660-664.

148. Mannavola D, Coco P, Vannucchi G, Coco P, Vannucchi G, Bertuelli R, Carletto M, Casali PG, Beck-Peccoz P, Fugazzola L 2007 A novel tyrosine-kinase selective inhibitor, sunitinib, induces transient hypothyroidism by blocking iodine uptake. J Clin Endocrinol Metab 92:3531-3534.

149. Wong E, Rosen LS, Mulay M, Vanvugt A, Dinolfo M, Tomoda C, Sugawara M, Hershman JM 2007 Sunitinib induces hypothyroidism in advanced cancer patients and may inhibit thyroid peroxidase activity. Thyroid 17:351355.

150. Shinohara N, Takahashi M, Kamishima T, Ikushima $H$, Otsuka N, Ishizu A, Shimizu C, Kanayama H, Nonomura K 2011 The incidence and mechanism of sunitinib-induced thyroid atrophy in patients with metastatic renal cell carcinoma. Br J Cancer 104:241-247.

151. Baldazzi V, Tassi R, Lapini A, Santomaggio C, Carini M, Mazzanti R 2012 The impact of sunitinib-induced hypothyroidism on progression-free survival of metastatic renal cancer patients: a prospective single-center study. Urol Oncol 30:704-710.

152. Rutkowski P, Bylina E, Klimczak A, Switaj T, Falkowski S, Kroc J, Lugowska I, Brzeskwiniewicz M, Melerowicz W, Osuch C, Mierzejewska E, Wasielewski K, Woźniak A, Grzesiakowska U, Nowecki ZI, Siedlecki JA, Limon J 2012 The outcome and predictive factors of sunitinib therapy in advanced gastrointestinal stromal tumors (GIST) after imatinib failure-one institution study. BMC Cancer 12:107.

153. Sabatier R, Eymard JC, Walz J, Deville JL, Narbonne H, Boher JM, Salem N, Marcy M, Brunelle S, Viens P, Bladou F, Gravis G 2012 Could thyroid dysfunction influence outcome in sunitinib-treated metastatic renal cell carcinoma? Ann Oncol 23:714-721.

154. Tamaskar I, Bukowski R, Elson P, Ioachimescu AG, Wood L, Dreicer R, Mekhail T, Garcia J, Rini BI 2008 Thyroid function test abnormalities in patients with metastatic renal cell carcinoma treated with sorafenib. Ann Oncol 19:265268.

155. Clement P, Wolter P, Stefan C, Decallonne B, Dumez H, Wildiers H, Schöffski P 2008 Thyroid dysfunction in patients (pts) with metastatic renal cell cancer (RCC) treated 
with sorafenib. J Clin Oncol 26 (May 20 suppl):16145 (abstract).

156. Miyake H, Kurahashi T, Yamanaka K, Kondo Y, Muramaki M, Takenaka A, Inoue TA, Fujisawa M 2010 Abnormalities of thyroid function in Japanese patients with metastatic renal cell carcinoma treated with sorafenib: a prospective evaluation. Urol Oncol 28:515-519.

157. Di Lorenzo G, Cartenì G, Autorino R, Bruni G, Tudini M, Rizzo M, Aieta $M$, Gonnella $A$, Rescigno $P$, Perdonà $S$, Giannarini G, Pignata S, Longo N, Palmieri G, Imbimbo C, De Laurentiis M, Mirone V, Ficorella C, De Placido S 2009 Phase II study of sorafenib in patients with sunitinib-refractory metastatic renal cell cancer. J Clin Oncol 27:4469-4474.

158. Riesenbeck LM, Bierer S, Hoffmeister I, Köpke T, Papavassilis P, Hertle L, Thielen B, Herrmann E 2011 Hypothyroidism correlates with a better prognosis in metastatic renal cancer patients treated with sorafenib or sunitinib. World J Urol 29:807-813.

159. Schmidinger M, Vogl UM, Bojic M, Lamm W, Heinzl H, Haitel A, Clodi M, Kramer G, Zielinski CC 2011 Hypothyroidism in patients with renal cell carcinoma: blessing or curse? Cancer 117:534-544.

160. Kitajima K, Takahashi S, Maeda T, Yoshikawa T, Ohno Y, Fujii M, Miyake H, Fujisawa M, Sugimura K 2012 Thyroid size change by $\mathrm{CT}$ monitoring after sorafenib or sunitinib treatment in patients with renal cell carcinoma: comparison with thyroid function. Eur J Radiol 81:2060-2065.

161. Clemons J, Gao D, Naam M, Breaker K, Garfield D, Flaig TW 2012 Thyroid dysfunction in patients treated with sunitinib or sorafenib. Clin Genitourin Cancer 10:225-231.

162. Feldt S, Schüssel K, Quinzler R, Franzmann A, Czeche S, Ludwig WD, Schulz M 2012 Incidence of thyroid hormone therapy in patients treated with sunitinib or sorafenib: a cohort study. Eur J Cancer 48:974-981.

163. Zhao J, Zhu Y, Zhang C, Wang X, He H, Wang H, Wu Y, Zhou W, Shen Z 2012 Sorafenib or sunitinib as postoperative adjuvant therapy for Chinese patients with locally advanced clear cell renal cell carcinoma at high risk for disease recurrence. Urol Oncol May 30; DOI:10.1016/j.urolonc.2012 .04 .019

164. Mukohara T, Nakajima H, Mukai H, Nagai S, Itoh K, Umeyama Y, Hashimoto J, Minami H 2010 Effect of axitinib (AG-013736) on fatigue, thyroid-stimulating hormone, and biomarkers: a phase I study in Japanese patients. Cancer Sci 101:963-968.

165. Fujiwara Y, Kiyota N, Chayahara N, Suzuki A, Umeyama Y, Mukohara T, Minami H 2012 Management of axitinib (AG013736)-induced fatigue and thyroid dysfunction, and predictive biomarkers of axitinib exposure: results from phase I studies in Japanese patients. Invest New Drugs 30:1055-1064.

166. Tomita $Y$, Uemura H, Fujimoto H, Kanayama HO, Shinohara N, Nakazawa H, Imai K, Umeyama Y, Ozono S, Naito S, Akaza H; Japan Axitinib Phase II Study Group 2011 Key predictive factors of axitinib (AG-013736)-induced proteinuria and efficacy: a phase II study in Japanese patients with cytokine-refractory metastatic renal cell Carcinoma. Eur J Cancer 47:2592-2602.

167. Rini BI, Wilding G, Hudes G, Stadler WM, Kim S, Tarazi J, Rosbrook B, Trask PC, Wood L, Dutcher JP 2009 Phase II study of axitinib in sorafenib-refractory metastatic renal cell carcinoma. J Clin Oncol 27:4462-4468.

168. Rini BI, Escudier B, Tomczak P, Kaprin A, Szczylik C, Hutson TE, Michaelson MD, Gorbunova VA, Gore ME, Rusakov IG, Negrier S, Ou YC, Castellano D, Lim HY,
Uemura H, Tarazi J, Cella D, Chen C, Rosbrook B, Kim S, Motzer RJ 2011 Comparative effectiveness of axitinib versus sorafenib in advanced renal cell carcinoma (AXIS): a randomised phase 3 trial. Lancet 378:1931-1939.

169. Daimon M, Kato T, Kaino W, Takase K, Karasawa S, Wada K, Kameda W, Susa S, Oizumi T, Tomita Y, Kato T 2012 Thyroid dysfunction in patients treated with tyrosine kinase inhibitors, sunitinib, sorafenib and axitinib, for metastatic renal cell carcinoma. Jpn J Clin Oncol 42: 742-747.

170. Joensuu H, De Braud F, Grignagni G, De Pas T, Spitalieri G, Coco P, Spreafico C, Boselli S, Toffalorio F, Bono P, Jalava T, Kappeler C, Aglietta M, Laurent D, Casali PG 2011 Vatalanib for metastatic gastrointestinal stromal tumour (GIST) resistant to imatinib: final results of a phase II study. Br J Cancer 104:1686-1690.

171. Sherman SI, Wirth LJ, Droz JP, Hofmann M, Bastholt L, Martins RG, Licitra L, Eschenberg MJ, Sun YN, Juan T, Stepan DE, Schlumberger MJ 2008 Motesanib diphosphate in progressive differentiated thyroid cancer. N Engl J Med 359:31-42.

172. Blumenschein GR Jr., Kabbinavar F, Menon H, Mok TS, Stephenson J, Beck JT, Lakshmaiah K, Reckamp K, Hei YJ, Kracht K, Sun YN, Sikorski R, Schwartzberg L 2011 A phase II, multicenter, open-label randomized study of motesanib or bevacizumab in combination with paclitaxel and carboplatin for advanced nonsquamous non-small-cell lung cancer. Ann Oncol 22:2057-2067.

173. Sternberg CN, Davis ID, Mardiak J, Szczylik C, Lee E, Wagstaff J, Barrios CH, Salman P, Gladkov OA, Kavina A, Zarba JJ, Chen M, McCann L, Pandite L, Roychowdhury DF, Hawkins RE 2010 Pazopanib in locally advanced or metastatic renal cell carcinoma: results of a randomized phase III trial. J Clin Oncol 28:1061-1068.

174. Matulonis UA, Berlin S, Ivy P, Tyburski K, Krasner C, Zarwan C, Berkenblit A, Campos S, Horowitz N, Cannistra SA, Lee H, Lee J, Roche M, Hill M, Whalen C, Sullivan L, Tran C, Humphreys BD, Penson RT 2009 Cediranib, an oral inhibitor of vascular endothelial growth factor receptor kinases, is an active drug in recurrent epithelial ovarian, fallopian tube, and peritoneal cancer. J Clin Oncol 27:56015606.

175. Garland LL, Chansky K, Wozniak AJ, Tsao AS, Gadgeel SM, Verschraegen CF, Dasilva MA, Redman M, Gandara DR 2011 Phase II study of cediranib in patients with malignant pleural mesothelioma: SWOG S0509. J Thorac Oncol 6:1938-1945.

176. Asahina H, Tamura $Y$, Nokihara H, Yamamoto N, Seki $Y$, Shibata T, Goto Y, Tanioka M, Yamada Y, Coates A, Chiu YL, Li X, Pradhan R, Ansell PJ, McKeegan EM, McKee MD, Carlson DM, Tamura T 2012 An open-label, phase 1 study evaluating safety, tolerability, and pharmacokinetics of linifanib (ABT-869) in Japanese patients with solid tumors. Cancer Chemother Pharmacol 69:1477-1486.

177. Toh HC, Chen PJ, Carr BI, Knox JJ, Gill S, Ansell P, McKeegan EM, Dowell B, Pedersen M, Qin Q, Qian J, Scappaticci FA, Ricker JL, Carlson DM, Yong WP 2012 Phase 2 trial of Linifanib (ABT-869) in patients with unresectable or metastatic hepatocellular carcinoma. Cancer 2012 Jul 5. [Epub ahead of print]

178. Tan EH, Goss GD, Salgia R, Besse B, Gandara DR, Hanna $\mathrm{NH}$, Yang JC, Thertulien R, Wertheim M, Mazieres J, Hensing T, Lee C, Gupta N, Pradhan R, Qian J, Qin Q, Scappaticci FA, Ricker JL, Carlson DM, Soo RA 2011 Phase 
2 trial of Linifanib (ABT-869) in patients with advanced non-small cell lung cancer. J Thorac Oncol 6:1418-1425.

179. Weisberg E, Catley L, Wright RD, Moreno D, Banerji L, Ray A, Manley PW, Mestan J, Fabbro D, Jiang J, Hall-Meyers E, Callahan L, Della Gatta JL, Kung AL, Griffin JD 2007 Beneficial effects of combining nilotinib and imatinib in preclinical models of BCR-ABL+leukemias. Blood 109: 2112-2120.

180. Manley PW, Drueckes P, Fendrich G, Furet P, Liebetanz J, Martiny-Baron G, Mestan J, Trappe J, Wartmann M, Fabbro D 2010 Extended kinase profile and properties of the protein kinase inhibitor nilotinib. Biochim Biophys Acta 1804:445-453.

181. Kim TD, Schwarz M, Nogai H, Grille P, Westermann J, Plockinger U, Braun D, Schweizer U, Arnold R, Dorken B, le Coutre P 2010 Thyroid dysfunction caused by secondgeneration tyrosine kinase inhibitors in Philadelphia chromosome positive chronic myeloid leukemia. Thyroid 20: 1209-1214.

182. Salem AK, Fenton MS, Marion KM, Hershman JM 2008 Effect of sunitinib on growth and function of FRTL-5 thyroid cells. Thyroid 18:631-635.

183. Alexandrescu DT, Popoveniuc G, Farzanmehr H, Dasanu CA, Dawson N, Wartofsky L 2008 Sunitinib-associated lymphocytic thyroiditis without circulating antithyroid antibodies. Thyroid 18:809-812.

184. Kamba T, Tam BY, Hashizume H, Haskell A, Sennino B, Mancuso MR, Norberg SM, O'Brien SM, Davis RB, Gowen LC, Anderson KD, Thurston G, Joho S, Springer ML, Kuo CJ, McDonald DM 2006 VEGF-dependent plasticity of fenestrated capillaries in the normal adult microvasculature. Am J Physiol Heart Circ Physiol 290:H560-H576.

185. Rini BI, Tamaskar I, Shaheen P, Salas R, Garcia J, Wood L, Reddy S, Dreicer R, Bukowski RM 2007 Hypothyroidism in patients with metastatic renal cell carcinoma treated with sunitinib. J Natl Cancer Inst 99:81-83.

186. Makita N, Miyakawa M, Fujita T, Iiri T 2010 Sunitinib induces hypothyroidism with a markedly reduced vascularity. Thyroid 20:323-326.

187. Rogiers A, Wolter P, Op de Beeck K, Thijs M, Decallonne B, Schöffski P 2010 Shrinkage of thyroid volume in sunitinibtreated patients with renal-cell carcinoma: a potential marker of irreversible thyroid dysfunction? Thyroid 20: 317-322.

188. Hershman JM, Liwanpo L 2010 How does sunitinib cause hypothyroidism? Thyroid 20:243-244.

189. Reismuller B, Azizi AA, Peyrl A, Heinrich M, Gruber-Olipitz M, Luckner D, Rothschild KV, Slavc I 2010 Feasibility and tolerability of bevacizumab in children with primary CNS tumors. Pediatr Blood Cancer 54:681-686.

190. Willett CG, Boucher Y, di Tomaso E, Duda DG, Munn LL, Tong RT, Chung DC, Sahani DV, Kalva SP, Kozin SV, Mino M, Cohen KS, Scadden DT, Hartford AC, Fischman AJ, Clark JW, Ryan DP, Zhu AX, Blaszkowsky LS, Chen HX, Shellito PC, Lauwers GY, Jain RK 2004 Direct evidence that the VEGF specific antibody bevacizumab has antivascular effects in human rectal cancer. Nat Med 10:145-147.

191. Wang JF, Milosveski V, Schramek C, Fong GH, Becks GP, Hill DJ 1998 Presence and possible role of vascular endothelial growth factor in thyroid cell growth and function. J Endocrinol 157:5-12.

192. Sato K 2001 Vascular endothelial growth factors and thyroid disorders. Endocr J 48:635-646.
193. Ramsden JD 2000 Angiogenesis in the thyroid gland. J Endocrinol 166:475-480.

194. Makita N, Iiri T 2013 Tyrosine kinase inhibitor-induced thyroid disorders: a review and hypothesis. Thyroid 23:151-159.

195. de Groot JW, Zonnenberg BA, Plukker JT, van Der Graaf WT, Links TP 2005 Imatinib induces hypothyroidism in patients receiving levothyroxine. Clin Pharmacol Ther 78:433-438.

196. Dora JM, Leie MA, Netto B, Fogliatto LM, Silla L, Torres F, Maia AL 2008 Lack of imatinib-induced thyroid dysfunction in a cohort of non-thyroidectomized patients. Eur J Endocrinol 158:771-772.

197. Curran PG, DeGroot LJ 1991 The effect of hepatic enzymeinducing drugs on thyroid hormones and the thyroid gland. Endocr Rev 12:135-150.

198. Schröder-van der Elst JP, van der Heide D, DiStefano JJ 3rd, van der Bent C, Kaptein E, Visser TJ 1997 Effects of 5, 5'diphenylhydantoin on the metabolic pathway of thyroid hormone in rats. Eur J Endocrinol 136:324-329.

199. De Luca F, Arrigo T, Pandullo E, Siracusano MF, Benvenga S, Trimarchi F 1986 Changes in thyroid function tests induced by 2 month carbamazepine treatment in L-thyroxinesubstituted hypothyroid children. Eur J Pediatr 145: 77-79.

200. Takasu N, Takara M, Komiya I 2005 Rifampin induced hypothyroidism in patients with Hashimoto's thyroiditis. N Engl J Med 352:518-519.

201. de Groot JW, Links TP, van der Graaf WT 2006 Tyrosine kinase inhibitors causing hypothyroidism in a patient on levothyroxine. Ann Oncol 17:1719-1720.

202. Abdulrahman RM, Verloop H, Hoftijzer H, Verburg E, Hovens GC, Corssmit EP, Reiners C, Gelderblom H, Pereira AM, Kapiteijn E, Romijn JA, Visser TJ, Smit JW 2010 Sorafenib-induced hypothyroidism is associated with increased type 3 deiodination. J Clin Endocrinol Metab 95:3758-3762.

203. Gupta-Abramson V, Troxel AB, Nellore A, Puttaswamy K, Redlinger M, Ransone K, Mandel SJ, Flaherty KT, Loevner LA, O'Dwyer PJ, Brose MS 2008 Phase II trial of sorafenib in advanced thyroid cancer. J Clin Oncol 26:4714-4719.

204. Schlumberger MJ, Elisei R, Bastholt L, Wirth LJ, Martins RG, Locati LD, Jarzab B, Pacini F, Daumerie C, Droz JP, Eschenberg MJ, Sun YN, Juan T, Stepan DE, Sherman SI 2009 Phase II study of safety and efficacy of motesanib in patients with progressive or symptomatic, advanced or metastatic medullary thyroid cancer. J Clin Oncol 27:37943801.

205. Robinson BG, Paz-Ares L, Krebs A, Vasselli J, Haddad R 2010 Vandetanib $(100 \mathrm{mg})$ in patients with locally advanced or metastatic hereditary medullary thyroid cancer. J Clin Endocrinol Metab 95:2664-2671.

206. Kappers MH, van Esch JH, Smedts FM, de Krijger RR, Eechoute K, Mathijssen RH, Sleijfer S, Leijten F, Danser AH, van den Meiracker AH, Visser TJ 2011 Sunitinib-induced hypothyroidism is due to induction of type 3 deiodinase activity and thyroidal capillary regression. J Clin Endocrinol Metab 96:3087-3094.

207. Ohba K, Takayama T, Matsunaga H, Matsushita A, Sasaki S, Oki Y, Ozono S, Nakamura H 2013 Inappropriate elevation of serum thyrotropin levels in patients treated with axitinib. Thyroid 24:443-448.

208. Fonseca T, Medina M, Campos M, Wittmann G, MoraGarzon M, Ueta C, Arrojo e drigo R, Werneck-de-castro J, 
Caicedo D, Gereben B, Fekete C, Lechan R, Bianco AC 2012 Pituitary-specific inactivation of type II deiodinase (D2) reveals coordination between the conversion of $\mathrm{T} 4$ to $\mathrm{T} 3$ in thyrothrophs and in tanycytes in the T4-mediated TSH feedback mechanism. Thyroid 22:A10 (abstract).

209. Braun D, Kim TD, le Coutre P, Kohrle J, Hershman JM, Schweizer U 2012 Tyrosine kinase inhibitors noncompetitively inhibit MCT8-mediated iodothyronine transport. J Clin Endocrinol Metab 97:E100-E105.

210. Kliewer SA, Umesono K, Mangelsdorf DJ, Evans RM 1992 Retinoid X receptor interacts with nuclear receptors in retinoic acid, thyroid hormone and vitamin D3 signalling. Nature 355:446-449.

211. Keller H, Dreyer C, Medin J, Mahfoudi A, Ozato K, Wahli W 1993 Fatty acids and retinoids control lipid metabolism through activation of peroxisome proliferator-activated receptor-retinoid X receptor heterodimers. Proc Natl Acad Sci USA 90:2160-2164.

212. Duvic M, Hymes $K$, Heald $P$, Breneman D, Martin AG, Myskowski P, Crowley C, Yocum RC; Bexarotene Worldwide Study Group 2001 Bexarotene is effective and safe for treatment of refractory advanced-stage cutaneous T-cell lymphoma: multinational phase II-III trial results. J Clin Oncol 19:2456-2471.

213. Haugen BR 2009 Drugs that suppress TSH or cause central hypothyroidism. Best Pract Res Clin Endocrinol Metab 23:793-800.

214. Golden WM, Weber KB, Hernandez TL, Sherman SI, Woodmansee WW, Haugen BR 2007 Single-dose rexinoid rapidly and specifically suppresses serum thyrotropin in normal subjects. J Clin Endocrinol Metab 92:124-130.

215. Sherman SI, Gopal J, Haugen BR, Chiu AC, Whaley K, Nowlakha P, Duvic M 1999 Central hypothyroidism associated with retinoid $X$ receptor-selective ligands. $\mathrm{N}$ Engl J Med 340:1075-1079.

216. Duvic M, Martin AG, Kim Y, Olsen E, Wood GS, Crowley CA, Yocum RC; Worldwide Bexarotene Study Group 2001 Phase 2 and 3 clinical trial of oral bexarotene (Targretin capsules) for the treatment of refractory or persistent earlystage cutaneous T-cell lymphoma. Arch Dermatol 137:581593.

217. Sharma V, Hays WR, Wood WM, Pugazhenthi U, St. Germain DL, Bianco AC, Krezel W, Chambon P, Haugen BR 2006 Effects of rexinoids on thyrotrope function and the hypothalamic-pituitary-thyroid axis. Endocrinology 147: 1438-1451.

218. Liu S, Ogilvie KM, Klausing K, Lawson MA, Jolley D, Li D, Bilakovics J, Pascual B, Hein N, Urcan M, Leibowitz MD 2002 Mechanism of selective retinoid X receptor agonistinduced hypothyroidism in the rat. Endocrinology 143: 2880-2885.

219. Smit JW, Stokkel MP, Pereira AM, Romijn JA, Visser TJ 2007 Bexarotene-induced hypothyroidism: bexarotene stimulates the peripheral metabolism of thyroid hormones. J Clin Endocrinol Metab 92:2496-2499.

220. Howell SR, Shirley MA, Ulm EH 1998 Effects of retinoid treatment of rats on hepatic microsomal metabolism and cytochromes $\mathrm{P} 450$. Correlation between retinoic acid receptor/retinoid $\mathrm{x}$ receptor selectivity and effects on metabolic enzymes. Drug Metab Dispos 26:234-239.

221. O'Connor P, Feely J 1987 Clinical pharmacokinetics and endocrine disorders. Therapeutic implications. Clin Pharmacokinet 13:345-364.
222. Chen SY, Kao PC, Lin ZZ, Chiang WC, Fang CC 2009 Sunitinib-induced myxedema coma. Am J Emerg Med 27:370.e1-370.e3.

223. Collinson F, Vasudev N, Berkin L, Khan MM, Selby PJ, Brown JE 2011 Sunitinib-induced severe hypothyroidism with cardiac compromise. Med Oncol 28 (Suppl 1):S699_ S701.

224. Di Lorenzo G, Autorino R, Bruni G, Cartenì G, Ricevuto E, Tudini M, Ficorella C, Romano C, Aieta M, Giordano A, Giuliano M, Gonnella A, De Nunzio C, Rizzo M, Montesarchio V, Ewer M, De Placido S 2009 Cardiovascular toxicity following sunitinib therapy in metastatic renal cell carcinoma: a multicenter analysis. Ann Oncol 20:15351542.

225. Cristofanilli M, Yamamura Y, Kau SW, Bevers T, Strom S, Patangan M, Hsu L, Krishnamurthy S, Theriault RL, Hortobagyi GN 2005 Thyroid hormone and breast carcinoma. Primary hypothyroidism is associated with a reduced incidence of primary breast carcinoma. Cancer 103:11221128.

226. Angelousi AG, Anagnostou VK, Stamatakos MK, Georgiopoulos GA, Kontzoglou KC 2012 Mechanisms in endocrinology: primary HT and risk for breast cancer: a systematic review and meta-analysis. Eur J Endocrinol 166:373-381.

227. Nelson M, Hercbergs A, Rybicki L, Strome M 2006 Association between development of hypothyroidism and improved survival in patients with head and neck cancer. Arch Otolaryngol Head Neck Surg 132:10411046.

228. Hercbergs AA, Goyal LK, Suh JH, Lee S, Reddy CA, Cohen $\mathrm{BH}$, Stevens GH, Reddy SK, Peereboom DM, Elson PJ, Gupta MK, Barnett GH 2003 Propylthiouracil-induced chemical hypothyroidism with high-dose tamoxifen prolongs survival in recurrent high grade glioma: a phase I/II study. Anticancer Res 23:617-626.

229. Wolter P, Stefan C, Decallonne B, Dumez H, Bex M, Carmeliet P, Schöffski P 2008 Evaluation of thyroid dysfunction as a candidate surrogate marker for efficacy of sunitinib in patients [pts] with advanced renal cell carcinoma [RCC]. J Clin Oncol 26 (Suppl 15): (5126 abstract).

230. Yang JC, Hughes M, Kammula U, Royal R, Sherry RM, Topalian SL, Suri KB, Levy C, Allen T, Mavroukakis S, Lowy I, White DE, Rosenberg SA 2007 Ipilimumab [antiCTLA4 antibody] causes regression of metastatic renal cell cancer associated with enteritis and hypophysitis. J Immunother 30:825-830.

231. Agarwala SS, Ribas A 2010 Current experience with CTLA4-blocking monoclonal antibodies for the treatment of solid tumors. J Immunother 33:557-569.

232. Torino F, Corsello SM, Longo R, Barnabei A, Gasparini G 2009 Is hypothyroidism a clinically relevant toxicity of tyrosine kinase inhibitors? Thyroid 19:539-540.

233. Eisen T, Sternberg CN, Robert C, Mulders P, Pyle L, Zbinden S, Izzedine H, Escudier B 2012 Targeted therapies for renal cell carcinoma: review of adverse event management strategies. J Natl Cancer Inst 104:93-113.

234. Torino F, Corsello SM, Longo R, Barnabei A, Gasparini G 2009 Hypothyroidism related to tyrosine kinase inhibitors: an emerging toxic effect of targeted therapy. Nat Rev Clin Oncol 6:219-228.

235. Assaf C, Bagot M, Dummer R, Duvic M, Gniadecki R, Knobler R, Ranki A, Schwandt P, Whittaker S 2006 Minimizing adverse side-effects of oral bexarotene in cutaneous 
T-cell lymphoma: an expert opinion. Br J Dermatol 155:261266.

236. Sherman SI 2003 Etiology, diagnosis, and treatment recommendations for central hypothyroidism associated with bexarotene therapy for cutaneous T-cell lymphoma. Clin Lymphoma 3:249-252.

237. Yervoy (ipilimumab): serious and fatal immune-mediated adverse reactions. www.yervoy.com/hcp/rems Accessed April 13, 2013.

238. Illouz F, Laboureau-Soares S, Dubois S, Rohmer V, Rodien P 2009 Tyrosine kinase inhibitors and modifications of thyroid function tests: a review. Eur J Endocrinol 160:331-336.

239. Baskin HJ, Cobin RH, Duick DS, Gharib H, Guttler RB, Kaplan MM, Segal RL 2002 American Association of Clinical Endocrinologists medical guidelines for clinical practice for the evaluation and treatment of hyperthyroidism and hypothyroidism. Endocr Pract 8:457-469.

240. Surks MI, Ortiz E, Daniels GH, Sawin CT, Col NF, Cobin RH, Franklyn JA, Hershman JM, Burman KD, Denke MA, Gorman C, Cooper RS, Weissman NJ 2004 Subclinical thyroid disease: scientific review and guidelines for diagnosis and management. JAMA 291:228-238.

241. Cooper DS, Biondi B 2012 Subclinical thyroid disease. Lancet 379:1142-1154.

242. Garfield DH, Hercbergs A, Davis PJ 2007 Re: Hypothyroidism in patients with metastatic renal cell carcinoma treated with sunitinib. J Natl Cancer Inst 99:975-976.
243. Lin HY, Sun M, Tang HY, Lin C, Luidens MK, Mousa SA, Incerpi S, Drusano GL, Davis FB, Davis PJ 2009 L-Thyroxine vs. 3, 5, 3'-triiodo-L-thyronine and cell proliferation: activation of mitogen-activated protein kinase and phosphatidylinositol 3-kinase. Am J Physiol Cell Physiol 296:C980-C991.

244. Davis PJ, Davis FB, Lin HY, Bergh JJ, Mousa S, Hercbergs A, Fenstermaker RA, Ciesielski MJ 2007 Cell surface receptor for thyroid hormone and tumor cell proliferation. Exp Rev Endocrinol Metabol 1:753-761.

245. Hamnvik OP, Larsen PR, Marqusee E 2012 Response: Re: Thyroid dysfunction from antineoplastic agents. J Natl Cancer Inst 104:423.

246. Hercbergs AA, Garfield D, Ashur-Fabian O, Davis PJ 2012 Re: Thyroid dysfunction from antineoplastic agents. J Natl Cancer Inst 104:422-423.

Address correspondence to: Salvatore Maria Corsello, MD Endocrinology Unit Università Cattolica Via Federico Cesi 72-I-00193 Roma Italy

E-mail: corsello.sm@mclink.it 